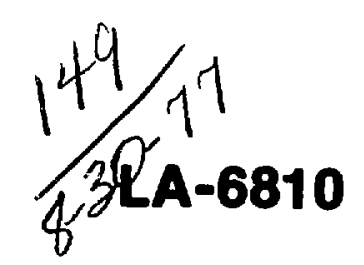

$D_{r} * 13.55$

UC-28

Issued: August 1977

\title{
High Resolution Spectrometer (HRS) Particle-Identification System
}

J. C. Pratt

J. E. Spencer

C. A. Whitten 


\section{CONTENTS}

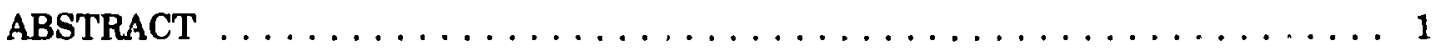

I. INTRODUCTION $\ldots \ldots \ldots \ldots \ldots \ldots \ldots \ldots \ldots \ldots \ldots \ldots \ldots$

II. DESCRIPTION OF THE SYSTEM $\ldots \ldots \ldots \ldots \ldots \ldots \ldots \ldots \ldots$

III. FUNCTIONS OF THE SYSTEM $\ldots \ldots \ldots \ldots \ldots \ldots \ldots \ldots \ldots \ldots \ldots$

A. Event Trigger System $\ldots \ldots \ldots \ldots \ldots \ldots \ldots \ldots \ldots \ldots \ldots \ldots \ldots$

B. Timing and Pulse-Size Information in the CAMAC System $\ldots \ldots \ldots \ldots \ldots 18$

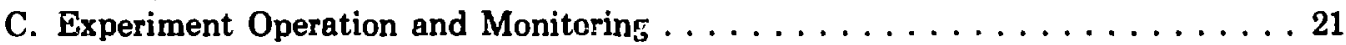

D. Cross-Section Normalization and Correction Information . . . . . . . . 22

IV. COUNTER CONSTRUCTION AND TESTING $\ldots \ldots \ldots \ldots \ldots \ldots \ldots 22$

V. COMPUTER INTERACTION WITH FAST ELECTRONICS $\ldots \ldots \ldots \ldots \ldots 23$

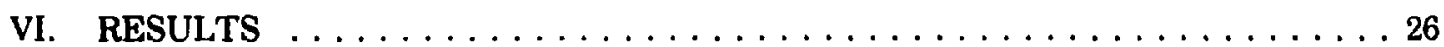

APPENIIX A. Counting-House Signals $\ldots \ldots \ldots \ldots \ldots \ldots \ldots \ldots \ldots \ldots \ldots \ldots \ldots$

APPENDIX B. Nonstandard Electronics $\ldots \ldots \ldots \ldots \ldots \ldots \ldots \ldots \ldots \ldots \ldots \ldots$

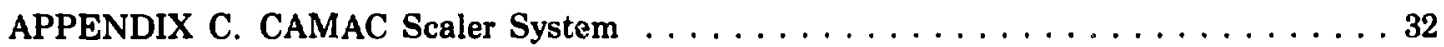

APPENDIX D. Computer Interaction with Experiment $\ldots \ldots \ldots \ldots \ldots \ldots \ldots$

APPENDIX E. Fast, Four-Channel Differential Discriminator $\ldots \ldots \ldots \ldots \ldots \ldots 35$

APPENDIX F. Pattern Coincidence/Recognition Module $\ldots \ldots \ldots \ldots \ldots \ldots \ldots$

APPENDIX G. Use of Cherenkov Counters for $(p, \pi)$ Experiments $\ldots \ldots \ldots \ldots \ldots 36$

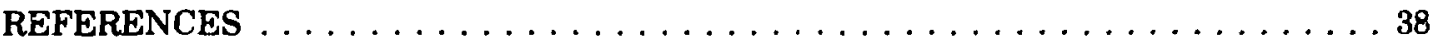

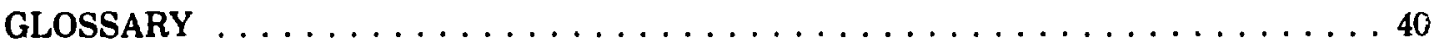

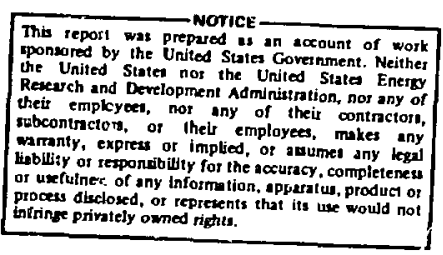




\title{
HIGH RESOLUTION SPECTROMETER (HRE) PARTICLE-IDENTIFICATION SYSTEM
}

\author{
by \\ J. C. Pratt \\ J. E. Spencer \\ C. A. Whitten
}

\begin{abstract}
The functions of the particle-identification system (PIDS) designed for the High Resolution Spectrometer facility (HRS) at LAMPF are described, together with the mechanical layout, counter hardware, and associated electronics. The system was designed for easy use and to be applicable to currently proposed experiments at HRS. The several strobe signals that can be generated correspond to different event types or characteristics, and logic configuration and timing can be remotely controlled by computer. Concepts of discrete pattern recognition and multidimensional, analog pulse discrimination are used to distinguish between different event types.
\end{abstract}

\section{INTRODUCTION}

The particle-identification system (PIDS) was designed to provide a variable, on-line definition of events-one that would optimize the data-taking capabilities of the High Resolution Spectrometer (HRS) facility. The following characieristics were considered in the design.

(1) Flexibility. The system should satisfy the requirements of a variety of experiments, yet require a minimum of physical modifications.

(2) Selectivity. The system should permit on-line selection of particle type and energy (e.g., the discrimination of quasi-elastic proton scattering from elastir. scattering).

(3) Comprehensiveness. For experiments with selective trigger requirements, the electronics shouid permit investigation of backgrounds and event losses with a minimum of time, effort, and difficulty.

(4) Efficiency. The system should give the experimenter the desireù events at the highest practical rate. Also, to maximize the amount of time available for data-taking, the system should have the ability to time and calibrate independently of the beam.

(5) Redundancy. The available data should have enough redundancy to permit useful analysis in the event of equipment failures or to provide internal consistency tests to insure proper operation of the equipment.

(6) Accuracy. The system should be able to monitor continuously the equipment status and function, as well as collect normalization information and associated corrections. 
For the vast amount of information one would like to access from a large system such as the HRS, data sampling and partition techniq ses are useful but are seldom satisfactory for logging primary events. The hardware, designed specifically for binary, on-line logical decisions and commurication with the com. puter, is probably the most critical part of the problem. Rather than put the burden on the software, one should phrase questions to the hardware in a logical yes-no format that requires the minimum of logical steps or sequential decisions to decide whether a significant event has occurred.

A significant event is one in which a subset of the available input variables assumes discrete, binary values that match one or more presettable paiterns. Inclusion of -edundancy in these patterns is based on the existence of what can be called "noise" in the system as we!! as nonindependence of the pattern variables. The latter limitation results from the character of the devices which are employed as well as physical limitations on their location and usage. The degree of redundancy is always arguable but is made variable so that the setup can be optimized for a particular experiment.

\section{DESCRIPTION OF THE SYSTEM}

Figure 1 shows a schematic of the QDMD spectrometer in the dispersion plane, together with the PIDS, beginning at the target and ending with the last Cherenkov counter. ${ }^{1}$ To the extent that spatial limitations admit, we have attempted to take optimal advantage of the spectrometer optics, which are point-to-point in the dispersion plane and pointto-point-to-parallel in the transverse plane with an intermediate crossover at the multipole element (M). The dashed elements in Fig. 1 indicate optional equipment which is not intended for initial operation. Virtually all of the experiments now under consideration can be done with the system defined by the solid-line elements, regardless of the characteristics of the incident beam.

The basic system consists of $J_{1,2}, W_{1,2}, W_{4,8}, S_{8,4}$, $S_{b}, C_{L}, S_{k}$, and $C_{D}$, in that order. $J_{1,2}$ is a pair of movable scintillator jaws which span the gap of the dipoles and which are remotely controllable from the Area $\mathrm{C}$ counting house (CCH) in exactly the same way as are the beam scanners in Beam Line $C$. Their longitudizal location in TRANSPORT

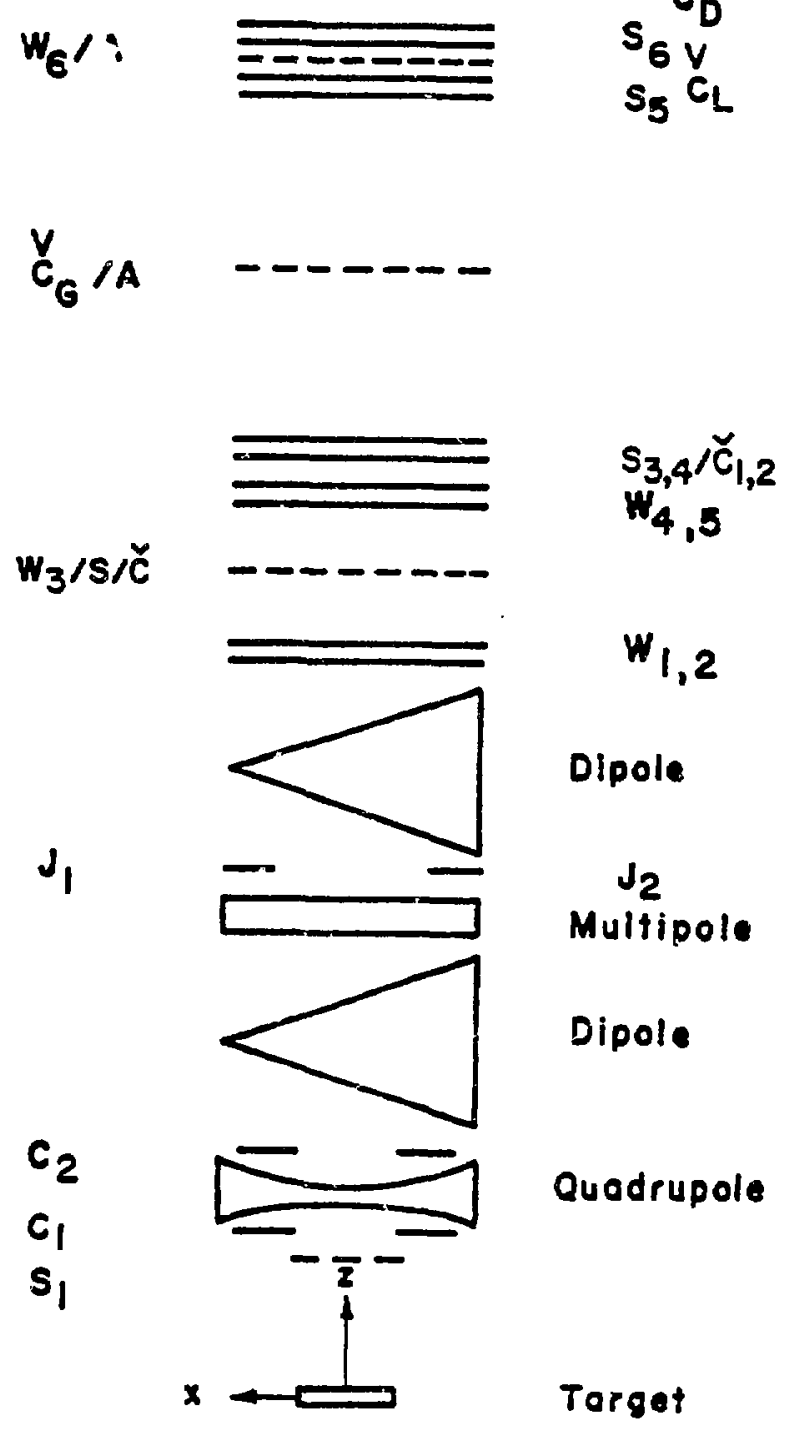

Fig. 1.

Schematic of the QDMD spectrometer and associated hardware, beginning at the target and ending with the NE 425 Cherenkov counter $\left(C_{D}\right)$. Dashed lines, described by the letters on the left, represent hardware that has not yet been built. $C_{1} \equiv i^{\text {th }}$ fixed collin ator for solid. angle limitation; $S_{\mathfrak{l}} \equiv \mathrm{i}^{\text {th }}$ plastic scintillation counter for coincidence; $J_{1}=i^{\text {th }}$ plastic scintillator jaw for solid-angle definition; $W_{1} \equiv$ multiwire proportional plane for track localization; $\breve{C} \equiv$ threshold Cherenkov counter, either gas (G), UVT Lucite (L), or NE 425 (D); $A=$ adjustable thickness absorber for varying particle velocity and range. 
notation ${ }^{2}$ is at a position where $(\mathrm{x} \mid \theta)$ is a maximum and $(x \mid \delta)$ is still comparatively small. As a result, their separation, together with the collimators $C_{1}$ and $C_{2}$, defines the angular acceptance in the plane transverse to the scattering plane. These jaws are also used as beam scanners in spectrometer tuneup and may be used as test counters for the PIDS or as coincidence counters to extend the available flight path when highest resolving power is not important.

Tile two sets of multiwire proportional chamber planes $W_{1,2}$ and $W_{1,8}$ are identical assemblies with a mean separation of $\sim 2 \mathrm{~m}$. Recause of their lengths transverse to the beam, all of the wires have been wound at $45^{\circ}$ to the dispersion plane and all have individual araplifiers. The sensitive area of these planes is about $10 \mathrm{~cm} \times 88 \mathrm{~cm}$ and since the gaip height of the dipoles is $10 \mathrm{~cm}$, the planes limit only the momentum acceptance of the spectrometer $(\delta \mathrm{p} / \mathrm{p} \leqslant \pm 2 \%)$. Each set determines $\mathrm{x}$ and $\mathrm{y}$ at its mean location and together the sets can be used to define $(x, y, \theta, \phi)$ along the focal surface of the spectrometer to within errors associated with the multiple scattering and the $1-\mathrm{mm}$ intrinsic resolution of the chambers. As a result, the sets provide a fixed reference relative to the magnets and give high count-rate capability and large momentum acceptance. The Brookhaven group that designed and built these counters will describe the system in detail in another report.

The coincident scintillator pairs $S_{8,4}$ and $S_{8,6}$ determine the flight time over their separation distanie which can be varied by moving $S_{6,0}$ up to a

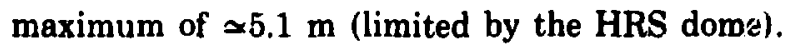
The scintillator pairs also provide pulse-height information for independent or redundant particleidentification purposes. The counters are thick enough to provide good time resolution for the lengths involved but not so thick as to make multiple scattering (or large neutron efficiencies) a significant problem for most of the particies and energies of interest. As one can see from the electronics layout in the next section, the number of scintillators need not be doubled, but doubling reduces false triggers from neutrons or other backgrounds by at least a factor of $10^{3}$ to $10^{4}$, depending on the experiment. Because increased flight paths mean increased counter size (in the present context), the outer counters are of a size applicable to all approved experiments. Obviously, this could lead to count-rate problems. Background sources include the beam dump, the beam-line plumbing, and the target area with its associated beam monitors which intercept the beam during an experiment.

The beam dump and beam plumbing are sized to permit a total beam loss of $<0.1 \%$ during an experiment on a $50-\mathrm{mg} / \mathrm{cm}^{2}$ target of ${ }^{250} \mathrm{U}$ and a beam dispersion at the target of $40 \mathrm{~cm} / \%$ with a $\pi / 3 \mathrm{~cm}-\mathrm{mr}$ phase space beam. The beam dump is a continuous flow water tank $>2 \mathrm{~m}$ long and $>30 \mathrm{~m}$ downstream of the target. Water helps to minimize neutron production as well as improve moderation of any neutrons that are produced. Aside from the target, the only material that always intercepts the beam inside the domed area of Fig. 2 is a dual secondary emission monitor (SEM) for beam-current integration $3.5 \mathrm{~m}$ downstream of the target when the spectrometer is at $\theta_{\mathrm{L}} \gtrsim 10^{\circ}$. The effective thickness of the SEM is $\leq 20 \mathrm{mg} / \mathrm{cm}^{2}$ of aluminum. Background from the target or SEM is unavoidable but both target and SEM can be shielded when necessary. The major source of background will then be the beam plumbing, which can produce enormous backgrounds when the magnets or the beam change. Monitoring of the single rates in the $S$ counters may then be one of the most sensitive indications of such changes.

The $S_{\text {s.4 }}$ pair serves to define the acceptance solid angle in the scattering plane via independent motion in the transverse directions. This pair also allows selective reduction of the energy acceptance over the length of the focal surface by again providing independent motion perpendicular to the optic axis, along the dispersion direction of the spectrometer.

The degrader and Cherenkov combinations permit a degree of discrimination between particles on the basis of their velocities. These elements add a simple and fast logical requirement to the event definition and should improve the on-line rejection ratio for some low cross-section measurements or for others where there is a comparatively poor signal-tonoise ratio and background suppression is important.

Figure 2 is a sectional view of the overall PIDS when the spectrometer is at zero degrees. Although the vertical setup of the HRS has a number of advantages, insofar as items such as $S_{b, 0}$ are concerned 


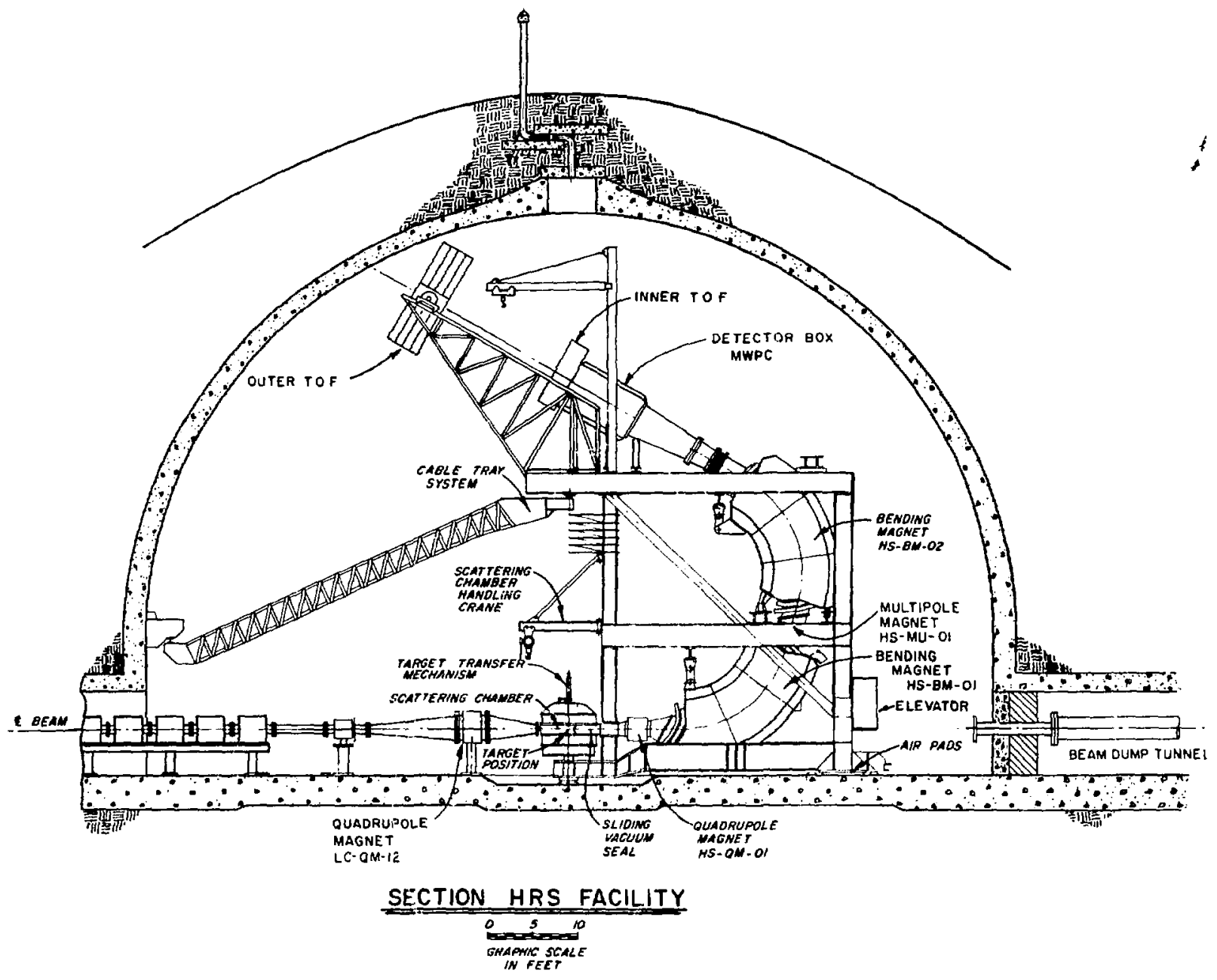

Fig. 2.

Elevation view of the spectrometer when the scattering angle is $\theta_{\mathrm{L}}=0^{\circ}$.

it is inconvenient to change detectors. In such a setup, reliability, flexibility, and comprehensiveness all contribute to the system's efficiency. A minimai layout was built and tested with the first available beam through the spectrometer. Subsequent sections describe the basic system and its associated electronics in more detail, as well as our initial experience with it.

\section{FUNCTIONS OF THE SYSTEM}

The particle identification hardware and electronics should:
- Provide strobe signals to chambers (MWPCstrobe) and CAMAC (CA.MAC-strobe),

- Provide timing and pulse-height information via the CAMAC system,

- Provide a variety of information to the experimenter on the setup and conduct of the experiment, and

- Provide cross-section normalization and correction information.

For efficient data acquisition the first item requires some form of event selection before acquisition. The second item is used for more precise software separation of desired events from background processes as well as for some redundancy. The third 
item helps to insure valid data, and the fourth provides absolute cross sections corrected for system deadtime and solid angle.

Figures 3 and 4 show the electronics layout at the location of the counters on the third level of the spectrometer and in the $\mathrm{CCH} \sim 100 \mathrm{~m}$ of signal path-length away. As discussed below, Fig. 3 could be substituted for Fig. 4 because all the necessary basic signals are routed to the $\mathrm{CCH}$. The modules, as well as their interconnections, depend somewhat on the available electronics and to some extent on the particular experiment. They will probably be modified as operating experience develops. For instance, the layout shown in Figs. 3 and 4 handles the basic configuration of Fig. 1 but is also suggestive of an $\mathrm{S}_{8} \mathrm{~S}_{6} \mathrm{C}_{6} \mathrm{~S}_{8} \mathrm{~S}_{6} \mathrm{~A}\left(\mathrm{C}_{\mathrm{L}}\right.$ or $\left.\mathrm{C}_{\mathrm{D}}\right)$ arrangement. More specific details concerning the layout of Fig. 3 are provided in Appendixes A and B.

\section{A. Event Trigger System}

The electronics that provide strobe signals to the chamber and to CAMAC are located on the frame of the spectrometer (Fig. 3) near the inner time of flight (TOF) $\left(S_{\mathbf{3}, \mathbf{s}}\right)$. The discriminator thresholds and pulse widths, as well as the logic configurations for some of the coincidence units, can be remotely programmed from the $\mathrm{CCH}$. The timing delays indicated in Fig. 3 are also remotely controllable as well as programmabla from the $\mathrm{CCH}$ using two specially designed modules." These features should maximize data output by minimizing the time required to make a decision on an event and by minimizing the time required to set the system up for any particular experiment (see Ref. 3 for details).

The trigger is selective in that it can distinguish between different particle types. When carried out external to the computer this feature can be difficult and error-prone and may require separate examination for each experiment. The data used in the basic PID system include:

- Flight time,

- Pulse height,

- Absorber range, and

- Velocity selection.

We call the overall setup a particle-identification system instead of a time-of-flight system because it is hard to say which of the above data are most im- portant in a configuration that handles a broad range of experiments (see Table I).

1. Timing. Timing is a powerful tool for particle separation in some experiments or in the computer but is not always adequate for the external trigger, as one can see from Fig. 5. The reason is that the computer numbers relate to the leading edge of the signals, whereas the overlap coincidences of the trigger system are subject to errors associated with the full pulse widths. There are, of course, obvious cases, such as separation of alphas and deuterons, in which even the computer cannot separate events solely on the basis of timing. Similarly whenever there is more than one kind of event trigger there may not be a time trigger at the $99 \%$ or even the $50 \%$ confidence level for all event types. There is also a time-resolution, energy-resolution "uncertainty principle" that can be quite limiting for highresolution systems like the HRS. Thus, although better external electronic timing schemes can be envisaged, most of them would be better implemented in the computer.

Several alternative timing schemes for the external trigger electronics are shown in Fig. 3 , but the basic system uses anode signals (LRS 612 amplifiers are optional) from the $S_{\delta, d} / S_{b, 0}$ pairs, with leadingedge discriminators of the LRS 621 BLP type (programmable). Scintillators comparable in thickness and light-collection solid angles to $S_{b, \theta}$ give a time resolution for $99 \%$ of the two counter coincidences of $\pm 2 \mathrm{~ns}$ for localized cosmic rays in the HRS setup with 12-cm tubes. Construction and testing of the specific hardware are described later. Here we discuss the basic time resolution achievable with the setup shown in Figs. 3 and 6.

If the timing for zero time difference between tubes 1 and 2 on any detector is based on the particle paths shown in Fig. 6, assumption of the AND mode for the two phototube signals from any scintillator $(n=1.58)$ and a propagation time in the scintillator of $\simeq 5$ to $8 \mathrm{~ns} / \mathrm{m}$ implies an additional variation of 0 to $4.5 \mathrm{~ns}$ from lack of track localization in the individual scintillators as well as the four counter coiricidences. The difference in particle paths between scintillators 3 and 6 is neglizible, with the worst case coming from $a \leqslant 75-\mathrm{cm}$ path difference in the scintillator. An overlap of $\gtrsim 2 n s$ to 


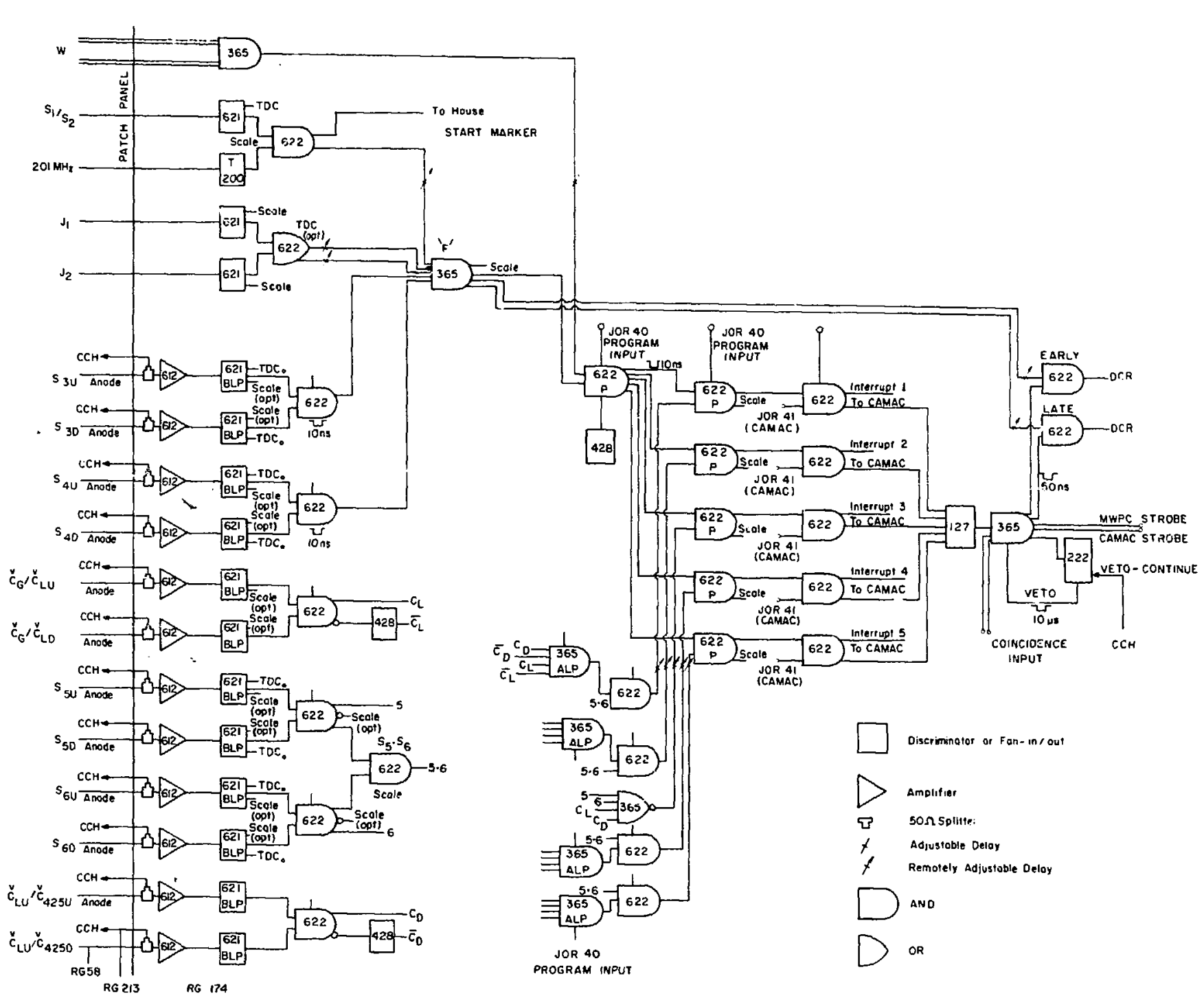

Fig. 3.

HRS fast electronics, located on top of the spectrometer, provide the particle-selection signal for the MWPC strobe. These modules allow control of thresholds, logic functions, delay times, and pulse widths from the $\mathrm{CCH}$. 


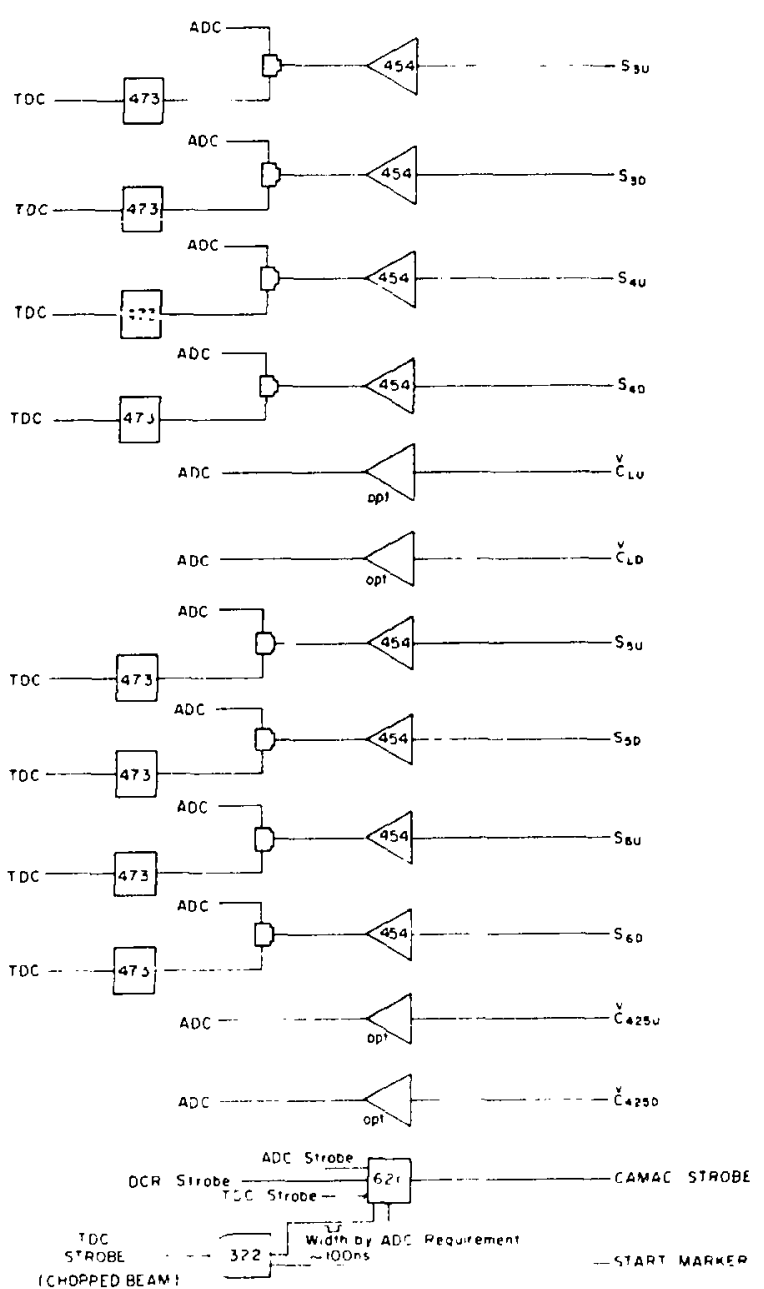

Fig. 4.

CCH electronics which can be used to supply independent timing and pulse-height signals to $C A M A C$.

generate the single-counter pulse then implies a minimum possible pulse width of $\gtrsim 7 \mathrm{~ns}$, vith $11 \mathrm{~ns}$ the recommended minimum for individual tube outputs from the nuter detectors whenever the full momentum acceptance is used.

The overall situation, including the contamination from another event type, can be seen in Fig. 7, where we have adjusted the time difference between $S_{3,4}$ and $S_{6,8}$ by 2 ns from its nominal value as determined by the assumed two-counter coincidence resolution. A 2-ns minimum overlap for unwanted background coincidences implies that we will start picking them up when $\Delta t \leqslant 7+4-2=9$ ns. A recommended 10-ns minimum pulse width out of the two-counter coincidence modules increases this to $12 \mathrm{~ns}$ so that we cannot separate al, particle types over the full momentum range of the HRS unless we add more electronics. Below $1 \mathrm{GeV} / \mathrm{c}$ we do much better, especially if the incidence of ${ }^{\mathrm{S}} \mathrm{He}$ is small so that background contamination for final state proton experiments would be small.

Other possible timing schemes require virtually no change in the electronics. Running in the OR mode for the two phototube signals from each scintillator increases the noise and does not improve the timing situation. Other alternatives to the basic system (Fig. 3) include "masking" segments of the wire-chamber output and/or reducing the energy acceptance defint $d$ hy the $S_{3,4}$ pair to reduce the effect from nonlocalized tracks. Addition of another level of logic would also help, e.g.,

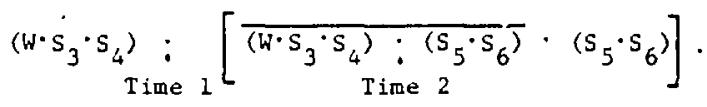

In effect, a coincidence is set for a specific particle type ( $\equiv$ time 1 ) with an additional coincidence set for another time ( $\equiv$ time 2) which detects signals that started earlier or later than valid time-1 signals. Again, event losses and backgrounds should be examined.

The LRS 624 Meantimer device can improve the situation because it can replace the LRS 622 coin. cidence units, periorming the AND for the phototubes on the two ends of a counter. The output is time-referenced to the midpoint of the counter in 1 -ns steps so that the time variation due to location is $\pm 0.5 \mathrm{~ns}$. To oversimplify, it replaces the ordinary two-phototube coincidence for each scintillator and forms the coincidence output when two signals meet after entering at opposite ends of a delay line. Signals arriving simultaneously at the Meantimer will overlap at the middle of the delay-a signal that originates near one end of the scintillator arrives at the deiay line earlier but travels a longer path (or has a longer delay) equivalent to the longer scintillator path of the other phototube pulse. The LRS 624 module is not shown in Fig. 3, but we are evaluating one. It gives $\mathrm{a} \pm 0.5$-ns time resolution and should simplify the adjustment and setup 
TABLE I

\section{SOME PARAMETERS FOR THE HRS PIDS OVER THE POSSIBLE} RANGE OF INCIDENT PROTON ENERGIES

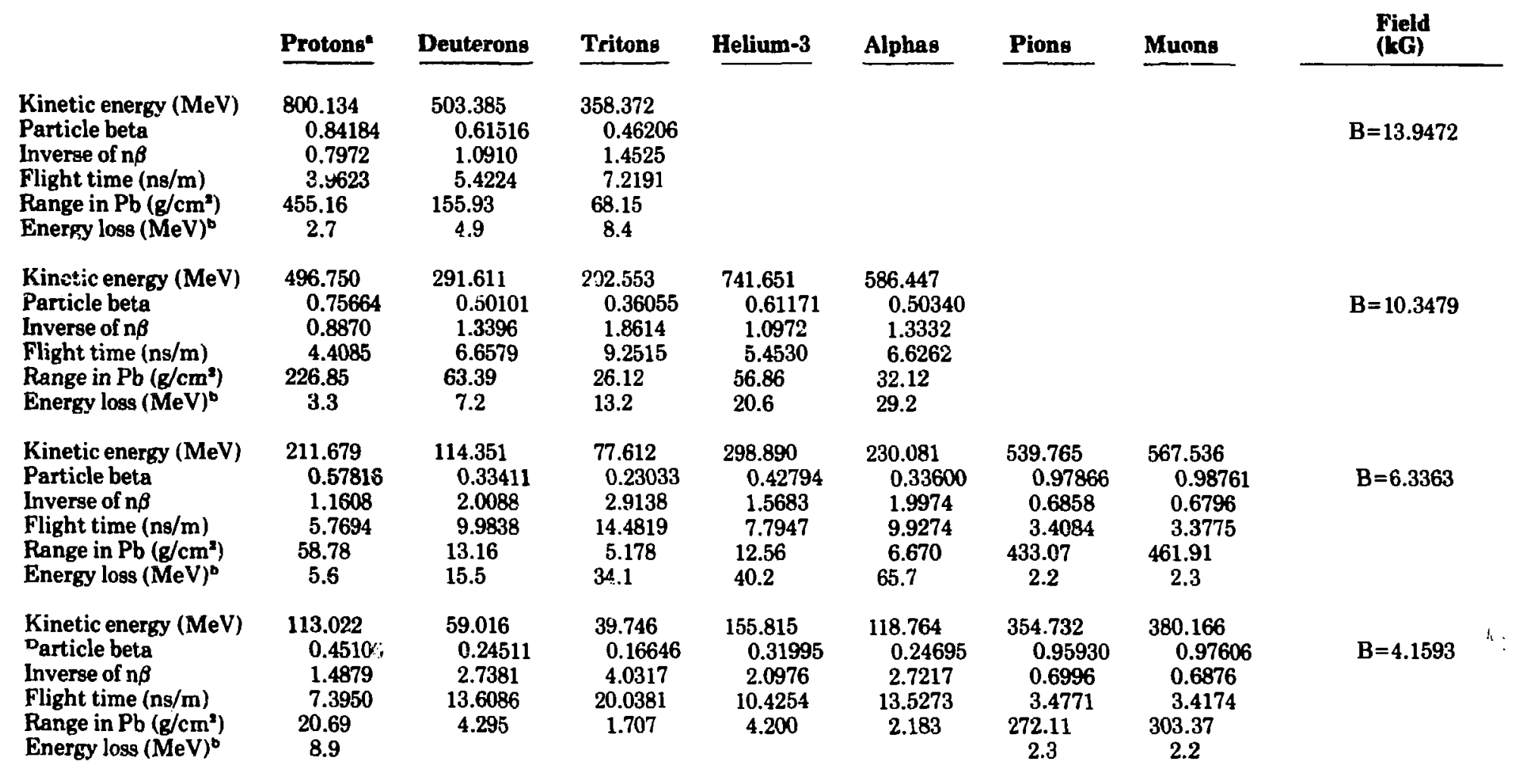

The specific proton energies correspond to nominal values available from the accelerator.

The energy loss is the most probable value predicted with the Vavilov distribution for a $1.27 \cdot \mathrm{cm}$ scintillator. 


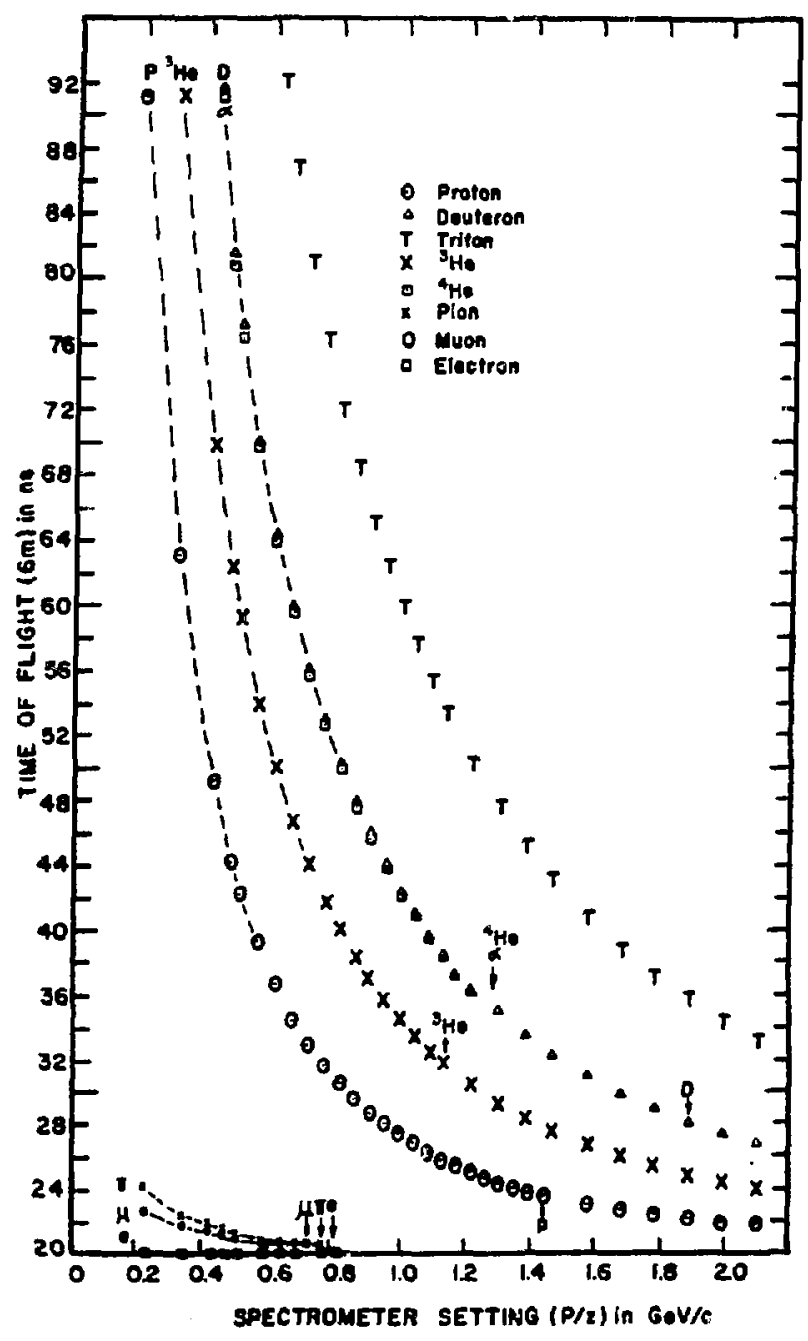

Fig. 5.

Time of flight for $6 \mathrm{~m}$ as a function of magnetic rigidity. Arrows mark the kinematic limits for the nroduction of each particle type by 800 . $\mathrm{MeV}$ protons.

procedure. Nevertheless, the 10-ns recommended minimum pulse width still seems valid.

Another possibility is to increase the flight times by replacing the $S_{\mathbf{3}, 4}$ pair, which provides the first. coincidence requirement, with $S_{1,2}$ or $J_{1,2}$. This can be accomplished remotely with the LRS 365 ALP majority coincidence unit (programmable), and if done carefully will nit appreciably degrade resolution.

A related timing scheme would involve use of a chopped beam so that these counters need not be in-

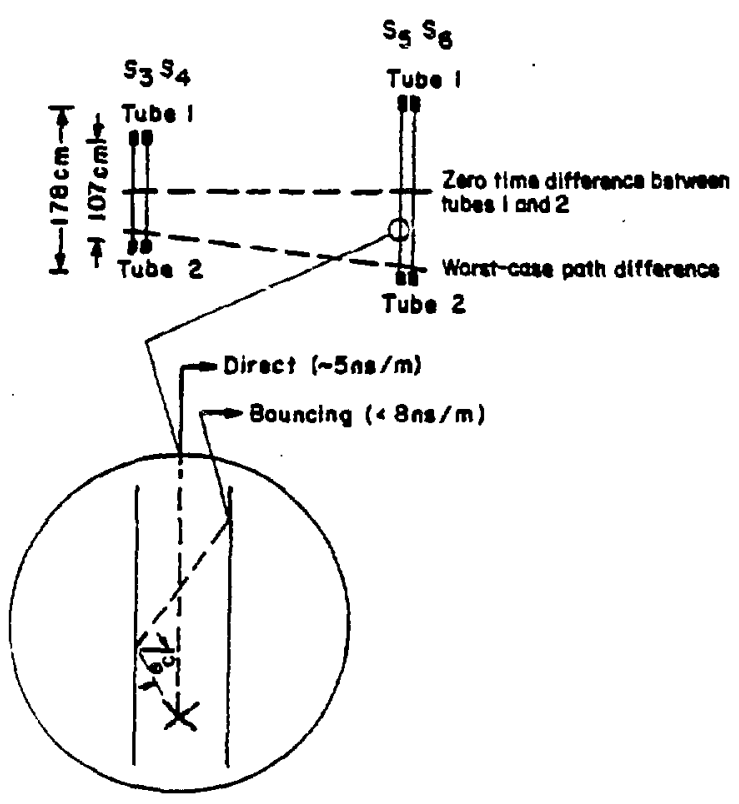

Fig. 6.

Counter sizes and conventions assumed. Photon travel time inside the scintillator between the particle track and the photomultiplier can vary the time of the photomultiplier output and the coincidence time between the two photomultipliers on each scintillator. Two paths (dashed lines) illustrate the extreme time variation for real particles from the spectrometer.

serted in the spectrometer. This option can be included in the external trigger in a number of ways, e.g., through use of the EGG T200/N fast discriminator which is specified to handle rates $>225$ MHz. The coincident scintillator(s) $S_{1,2}$ could be placed at $a$ forward angle in the scattering chamber, along the beam line, at the stripper, or wherever there are scintillation beam scanners. The only requirement is that they efficiently register the existence of beam. In Fig. 4, the TDC start for the internal analysis would also come from this signal or it could simply be run into another TDC channel to provide the basic time reference for the calculations. So far, 40 and 80 ns chopped beam have been used, i.e., one pulse in 8 or 16 wus allowed to pass, thereby decreasing the average available current by an order of magnitude or more.

If one used the inherent microstructure of the beam rather than dumping much of $i t$, proldems 


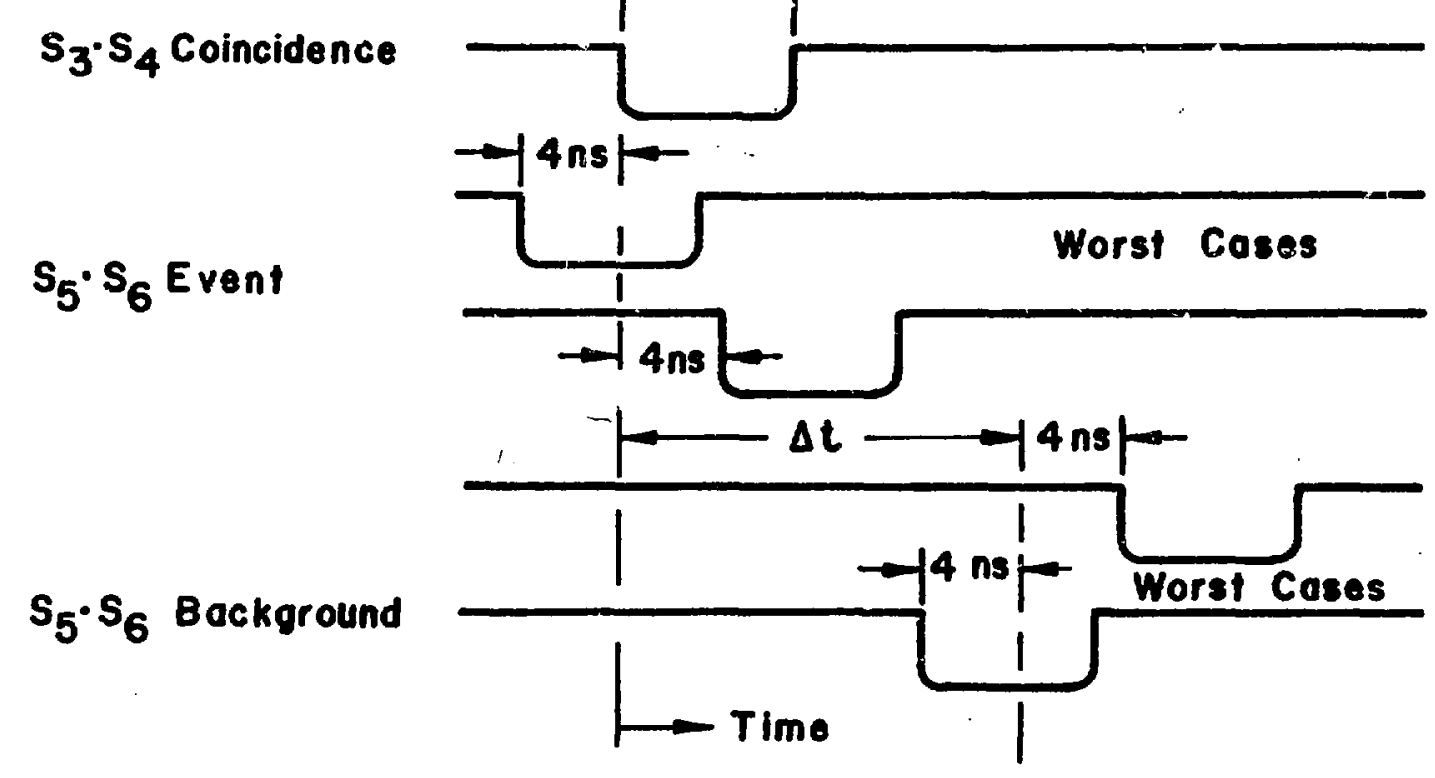

Fig. 7.

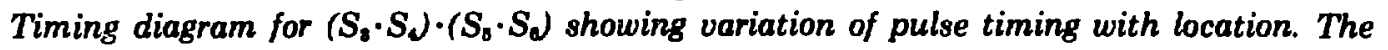
$S_{b} \cdot S_{0}$ coincidence timing is shifted from the flight time by half the time variation.

associated with the chopper would be eliminated. However, there is a fundamental limitation related to what we call the energy-time "uncertainty principle." To decrease the time resolution or uncertainty, $\Delta t_{\text {s, }}$ associated with a peak of width $\Delta E$, one must increase $\Delta \mathrm{E}$, and this increase has several effects. First, because the beam line has high resolution, the time uncertainty at the target will be greater than the raicropulse width from the accelerator $(\sim 1 / 4$ ns). Second, unless the spectrometer acceptance (much greater than the beam line) is not limited, the time uncertainty at the $S_{\mathbf{3}, 4}$ pair will be greater than the micropulse separation ( $~ 5 \mathrm{~ns}$ ).

To see how this can limit some of the possible timing schemes starting at the front end of the spectrometer, one can use first order TRANSPORT theory. ${ }^{2}$ In particular, the paraxial resolving power is directly proportional to the pulse length at the focal plane.

$R_{\mathrm{p}}=\frac{\mathrm{R}_{52}}{\mathrm{x}_{0}}=\frac{\left(l-l_{0}\right)}{\mathrm{x}_{0} \theta_{0}}$.
The monochromatic spot size at the target is $\mathbf{0 . 0 4}$ $\mathrm{cm}$ and the nominal angular divergence is $30 \mathrm{mr}$. Neglecting the pulse length at the target, we have

$R_{p}=\frac{R_{16}}{x_{0} R_{11}}= \pm 5.7 \cdot 10^{4}$

using the basic TRANSPORT numbers

$\mathbf{R}=\left[\begin{array}{rrrrrr}-0.8 & 0.0 & 0.0 & 0.0 & 0.0 & 18.3 \\ -4.0 & -1.2 & 0.0 & 0.0 & 0.0 & 16.0 \\ 0.0 & 0.0 & 0.1 & -0.2 & 0.0 & 0.0 \\ 0.0 & 0.0 & 5.2 & -0.1 & 0.0 & 0.0 \\ -5.9 & -2.3 & 0.0 & 0.0 & 1.0 & -12.3 \\ 0.0 & 0.0 & 0.0 & 0.0 & 0.0 & 1.0\end{array}\right]$

Thus, the inherent time resolution associated with a spectrometer setting of $1.4 \mathrm{GeV} / \mathrm{c}$ is:

$$
\begin{aligned}
& \Delta t_{p}-\left(R_{15} x_{\max }+R_{25} \theta_{\max }+l_{0}\right) / \beta c \\
& \leq \pm 4 \mathrm{~ns} \text { (protons) } \\
& \leq \pm 820 \text { (tritons) }
\end{aligned}
$$


and for the full momentum range $( \pm 2 \%)$ of the spectrometer:

$$
\begin{aligned}
\Delta t_{s} & \leqslant \pm 5 \text { ns (pr,tons) } \\
& \leqslant \pm 10 \text { ns (tritons). }
\end{aligned}
$$

This fundamental limitation can be reduced by restricting the acceptance of the spectrometer or by measuring the particle coordinates and correcting the measured times in a way similar to that for the resolution. Nonetheless, considering the $\simeq 22-\mathrm{m}$ target-to- $S_{2,4}$ distance, the method has value for the external strobe electronics when used with a chopped beam. The system was designed to handle most experiments without reliance on such beam characteristics for the same reason that the spectrometer was an energy-loss system rather than absolute energy, namely, inefficient use of beam current.

Among other timing alternatives that require changes in the basic electronics, one is the use of segmented counters for $S_{b}$ and/or $S_{B}$. Several phototubes would be distributed along the required length of scintillator rather than at either end. The increased phototubes and electronics, the timing required to set up the system, and the lack of unifor.. mity make it comparatively unattractive, consider. ing the intrinsic limitations on the external tims: resolution, unless count-rate problems cannot be adequately handled with shielding for the simpler two-tube counters.

A related alternative, possible with the system shown in Fig. 3, is a coarse-resolution ( $25-\mathrm{cm}$ ) wire chamber in the vicinity of $S_{8,0}$. The size and location of the active volume could be controlled from the $\mathrm{CCH}$, with the selected signals used to define the remotely programmable $W$ coincidence. The major advantage over "masking" segments of the $W_{1-4}$ chambers is that it allows investigation of largeangle scattering in the inner counters and its effect on the data obtained by the fast electronics. We suggest reducing the length of $S_{8,8}$ rather than using an additional counter in the vicinity of $W_{\mathrm{s}}$. Probably the simplest way to measure the efficiency of the system is to use the "pencil" beam to tune the beam line and spectrometer. However, this method would require a major modification of the electronics in the area of the $S_{b, 0}$ coincidence to prevent restriction of spectrometer acceptance, so it is not particularly useful for the external trigger.
We feel it is not worthwhile to improve the basic external timing technique, e.g., using zero-crossing or constant fraction or adding an external TAC and single-channel analyzer, unless analog circuitry for $\mathrm{dE} / \mathrm{dx}$ identification is added to the external trigger. This can be done in several ways and is discussed fully in Sec. III-B.

2. Pulse Height. The energy loss for different particles in a 1.3-cm scintillator is plotted in Fig. 8 as a function of spectrometer setting $(P / z)$. The points here are the most probable energy losses predicted by the Vavilov distribution." The 90 and $99 \%$ values are the "endpoint limit" on the integrals of the distribution on either sife of the most probable value. For this thickness, the asymmetry and spread of the energy loss are significantly different for the lighter particles. Figure $9 a$ gives the distributions for $530-\mathrm{MeV}$ protons incident on various thicknesses of scintillator and normalized to their most probable energy loss. Figure $9 \mathrm{~b}$ gives the corresponding distribution for alphas of the same $(\mathrm{P} / \mathrm{z})$ at $631 \mathrm{MeV}$, and Fig. 10 compares the energyloss distributions in a $1.3-\mathrm{cm}$ scintillator for the particle types of most interest at HRS.

The calculations were checked with a Monte Carlo ray-tracing code, ${ }^{\circ}$ which was also used to predict the plots for angular straggling shown in Fig. 11. The results can be related by $\theta \propto(Z / \beta) \sqrt{t}$, which is valid for thick targets according to Moliere theory. We chose the $530-\mathrm{MeV}$ proton energy because it is roughly centered on the working range of the HRS and corresponds to the highest available ${ }^{2} \mathrm{He}$ energy. Although the equilibrium charge for heavy ions passing through matter is generally a measured quantity relative to protons, the probability of having the maximum charge approaches one for $137 \beta / Z$ $\gtrsim 3$ (see Ref. 6). The importance of this effect (not included in the Vavilov calculations) is therefore negligible here if the numbers of Table I are used. As a result, we believe the predictions are valid and should provide some interesting comparisons to the results of measurements made with the system itself.

The measurements, of course, will include in:strumental effects which may be significant and require correction. In such cases, event determinations internal and external to the computer may differ considerably. On occasion the pulse size can be 


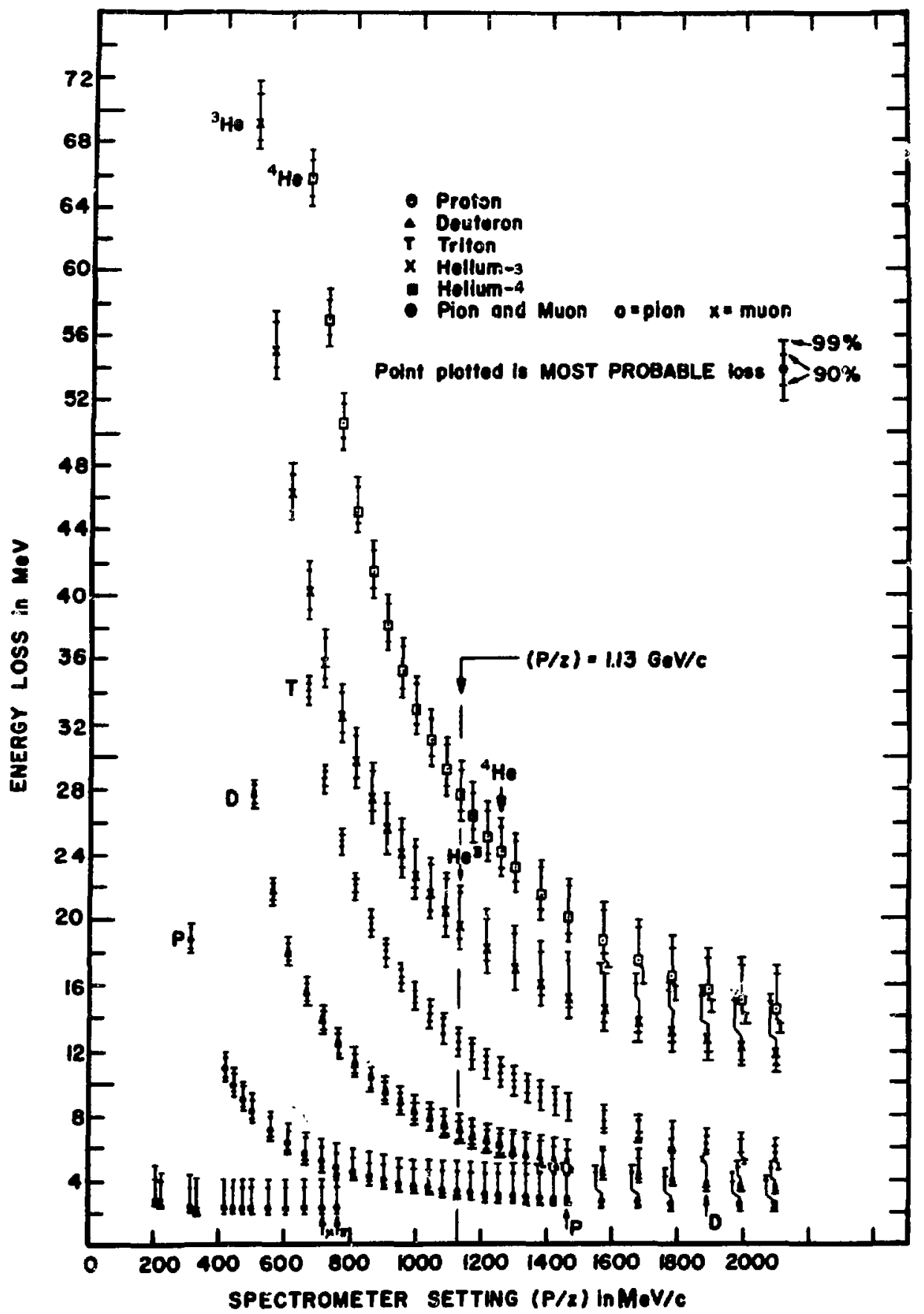

Fig. 3.

Energy loss in a 1.27-cm scintillator as a function of magnetic rigidity. Arrows mark the kinematic limits for production of different particle types by an $800-\mathrm{MeV}$ proton. The vertical dashed line at $1.13 \mathrm{GeV} / \mathrm{c}$ is the highest energy at ivhich we get all heavy particles and is used in a number of examples throughout the report. Photoelectron siatistics were not considered in this plot. 

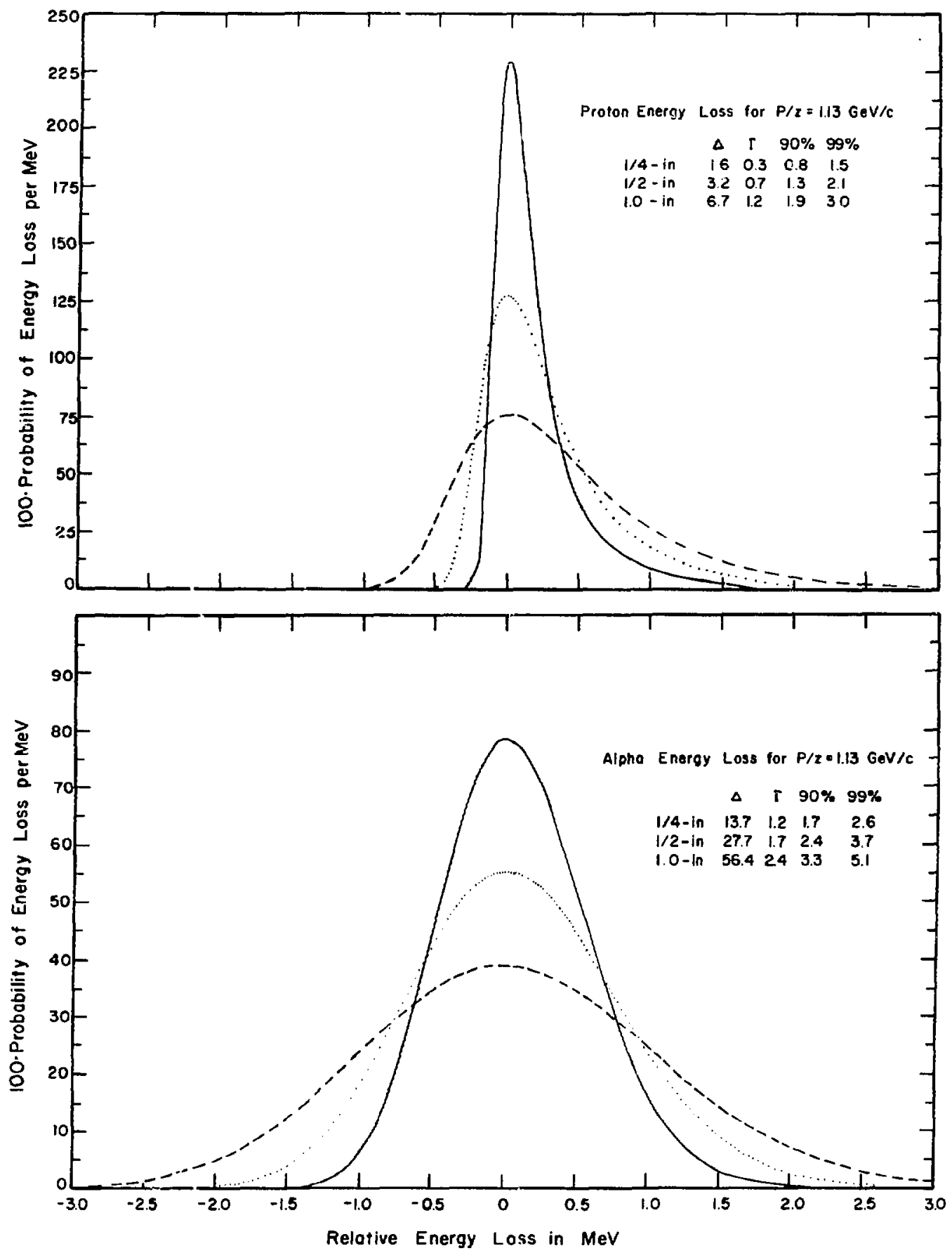

Fig. 9.

Variation in energy-loss distribution as a function of scintillation thickness. $\Delta$ is the mean energy loss, $\Gamma$ is the full width at half maximum, while the value labeled $90 \%$ is the separation between the $90 \%$ integrals starting from the location of the most probable energy loss. 


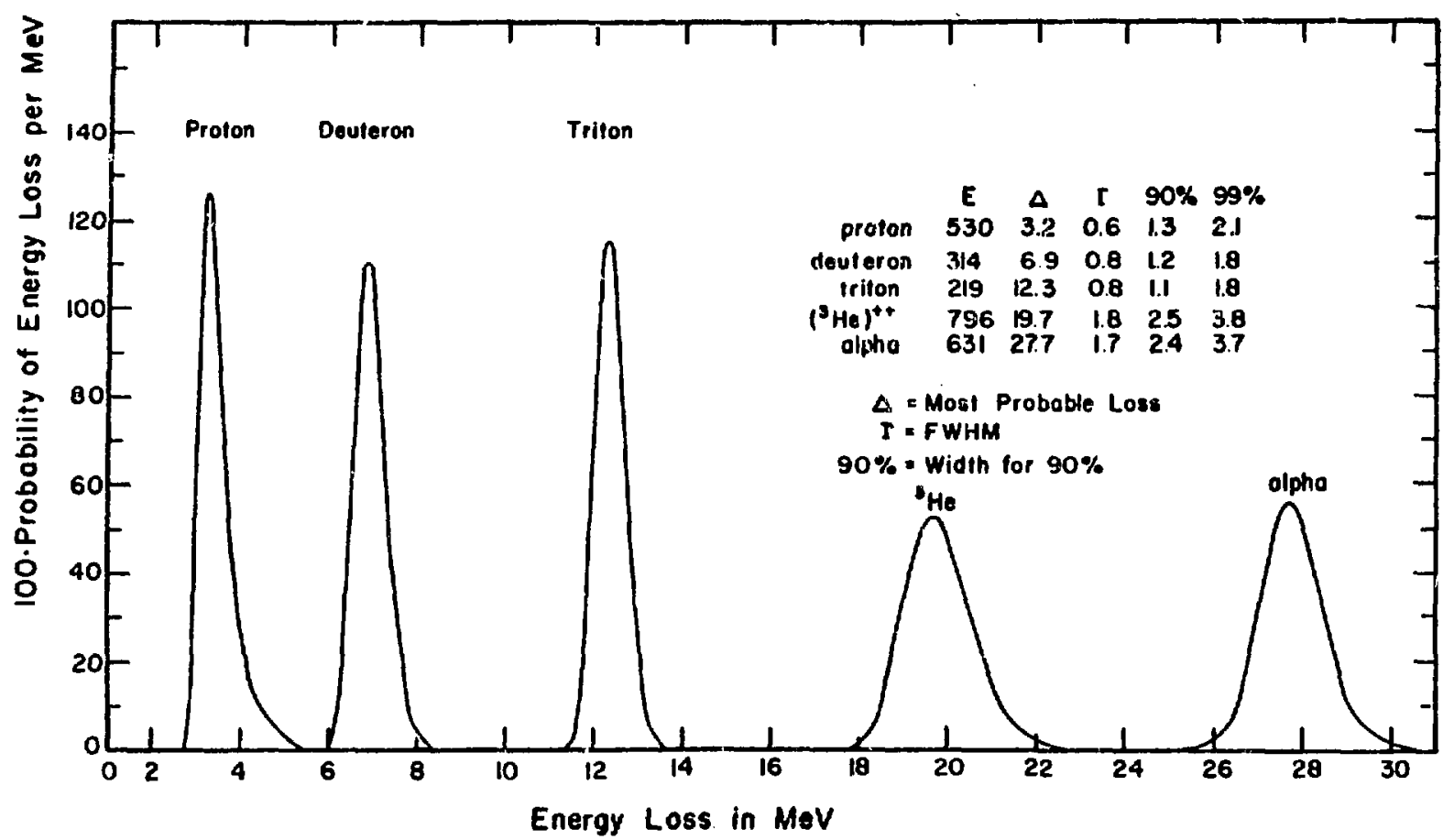

Fig. 10.

Calculated distribution of energy loss for $P / z=1.13 \mathrm{GeV} / \mathrm{c}$ in $1.27-\mathrm{cm}$-thick scintillator. (Based on Ref. 4.)

used to holp select particles with the fast electronics. Mor: often, calculations of the type shown argue that if large backgrounds do not threaten efficiency, the best place to deal with these data is in the computer.

The pulse sizes for each particle type have three primary sources of variation.

(1) Energy straggling as the particles traverse the scintillator,

(2) Attenuation from the collection path in the scintillator that may also affect the light-collection efficiency by reflection lusses, and

(3) Photoelectron statistics, which will reflect the photocathode conversion efficiency as well as photon-emission statistics.

The plots in Figs. 8-10 include only the first effect. The second effect influenced the design of the scintillators to the extent of

(1) Choice of low-attenuation phosphor (NE 110/Pilot Y),

(2) Use of twisted light pipes and $12-\mathrm{cm}$ photomultipliers, where necessary, to provide scintillator-areq-to-phototube-area matching, and

(3) Choice of thicker scintillator where multiple scattering permitted.

The last item nct only provided more absolute separation between energy losses for different particle types but also improved the photoelectron statistics. The increase in photoelectrons should also improve timing, ${ }^{7}$ but a slightly slower phosphor ( $\simeq 3 \mathrm{~ns}$ as opposed to $\simeq 2.5 \mathrm{~ns}$ for NE 102) and the 12 cm photomultiplier" were chosen more to benefit pulse-size separation than time resolution.

With the fast electronics, pulse-size separation can be achieved by setting diacriminator levels, but in using discriminator levels to select particle tyr og one must consider variations in pulse size. For an aitenuation $\simeq 50 \% / \mathrm{m}$, the tube at one end of a $2-\mathrm{m}$ scintillator sees the full light output for a particle at that end and $\approx 1 / 4$ the light output for the same event at the other end. By setting the electronics in the OR mode for each scintillator we can reduce the dynamic range that results from attenuation. When set to form a coincidence, the discriminator settings 


\section{$\int_{0}^{\theta} \theta_{\operatorname{mox}}$ Multiple Scattering .99}
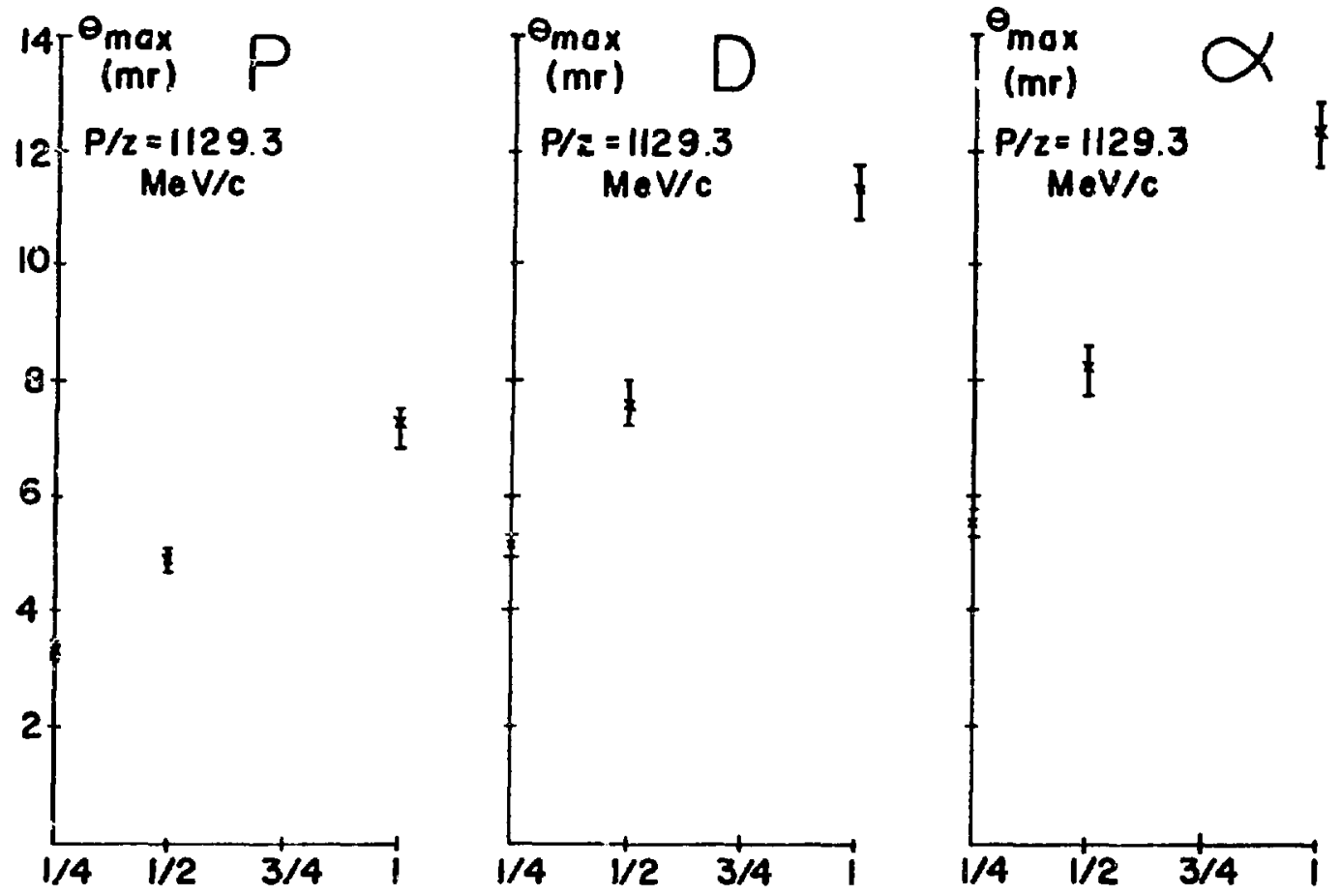

Scintillator Thickness $(\mathrm{cm} \times 2.5)$

Fig. 11.

Multiple scattering as a function of scintillator thickness for three types of particles at 1129.3 $\mathrm{MeV} / \mathrm{c}$. The scattering shown is the $99 \%$ point in the scattering angle distribution calculated by the SLTSCT code, with estimated errors for determining the $99 \%$ point.

must be low enough to allow detection over the entire range of signals or unacceptable efficiency problems result. In what follows, we assume that the scintillators are thick enough to make the signal from either phototube acceptable, and only half the length of the scintillator, with its attenuation, is considered.

A crude way of estimating the number of photoelectrons is discussed in the Particle Data Tables.' We assume a gamma for each $100 \mathrm{eV}$ deposited, $10 \%$ light-collection efficiency, $25 \%$ photoelectron response, and $60 \%$ light transmission ${ }^{10}$ for a $114-\mathrm{cm}$ length of $1.2 i-\mathrm{cm}$-thick scintillator. With $\approx 3.2 \mathrm{MeV}$ deposited by a 530 . $\mathrm{MeV}$ proton,

$N=\frac{3.2 \times 10^{6}}{100} \times 0.1 \times 0.60 \times 0.25 \simeq 500$.

To be conservative and to illustrate the effect on the calculations, take $10 \%$ of that number, $50 \pm 7$ photoelectrons. From Fig. 8, near the 'He kinematic limit, we similarly assume 


\section{$\mathbf{N} \pm \sqrt{\mathbf{N}} \quad \mathbf{N} \pm \sqrt{\mathbf{N}}$ \\ (at center) (at phototube)}

D (99\% upper limit) $\quad 125 \pm 11 \quad 250 \pm 16$

"Hè (99\% lower limit) $\quad 408 \pm 20 \quad 815 \pm 29$

A conservative estimate of the worst-case separation is then

D (99\% upper limit at phototube) $+3 \sqrt{\mathrm{N}} \cdot-298$ "He (99\% lower limit at center) $-3 \sqrt{\mathrm{N}}$---348

where the difference is $\approx 50$ or $\approx 12 \%$ of the worstcase "He lower limit. This difference can be enhanced by insertion of absorbers.

To choose only 'He by the pulse-height method one would cautiously raise the discriminator threshold to examine the event loss and resulting background of both protons and deuterons. To choose only $\mathrm{D}$, one could add a spare scintillation counter at the location of $C_{0}$ in Fig. 1. Figure 3 shows that this counter's coincidence or veto could be remotely selected, so a high discriminator setting could veto "He events. In fact, by the trivial operation of changing from normal to complementary outputs at the discriminator, any of the $S_{\mathbf{6}}-S_{6}$ could be used in this way with timing from the remaining counters. Helium-3 would also be greatly reduced or eliminated by this veto, leaving the timing for the $D$ selection unusually simple. Above $1 \mathrm{GeV} / \mathrm{c}$ one expects many more deuterons than any other composite particle so one would probably only be concerned with setting the discriminator thresholds and possibly adding absorber (see Appendix E).

Finally, because of the comparatively low energy of some of the particles of interest, we mention a technique common in low-energy experiments, which consists of a telescope of three detectors. Particles that stop in the second detector $\left(E_{2}\right)$ are identified by a circuit which multiplies the signal for a $\Delta E_{1}$ detector times the total energy signal $E=E_{2}+$ $\Delta E_{z}$. The method is based on the expression

$\frac{d E}{d x} \quad a \operatorname{cosst} \frac{x_{2}^{2}}{E} \log \left[\frac{2 m v^{2}}{1}\right]$

obtained by neglecting the weak energy dependence of the factor in brackets so that ( $\mathrm{dE} / \mathrm{dx}$ ) $\cdot \mathrm{E} \simeq$ const - $\mathrm{Mz}^{2}$ where $\mathrm{M}$ and $\mathrm{z}$ are the mass and charge of the incident particle. This method is attractive for its simplicity but its application to higher energy protons is virtually impossible. An altrernate possibility is based on the minimum pulse-height method" in which a minimal amount of material put in the particle's path produces a redundant set of pulses which essentially include that method as an important special case. The limited use of absorbers in this regard is discussed next.

3. Absorber. The precautions and benerits involved in the use of absorbers are familiar to every experimenter. Here we describe only the possibilities provided for in the basic system of Figs. 1-3.

The initial setup will have a "box" that can be placed anywhere in the stack of counters shown in Fig. 1. The box holds absorber sheets made of 1.6- to 6.4-inm-thick lead epoxied to aluminum backing ( $\$ 2.4 \mathrm{~mm}$ thick) and having a maximum load of 10 $\mathrm{cm}$ of lead. Minor changes in the electronics of Fig. 3 permit the trigger choices shown in Fig. 12, with a spare scintillator in place of $C_{L}$ and a second absorber box.

Also part of the basic system is the SLTSCT program," which can simulate the effects of a degrader on a beam of particles. This program can choose degrader and counter thicknesses and predict the sources of particle loss or background production.

4. Velocity Selection. The system shown in Fig. 1 includes two solid threshold Cherenkov counters. The first is a $2.54-\mathrm{cm}$-thick ultraviolet-transmitting (UVT) Lucite counter $\left(\mathrm{C}_{\mathrm{L}}\right)$. Its index of refraction is 1.49, which gives Cherenkuv light for $\beta=0.67$, corresponding to a proton kinetic energy of 330 $\mathrm{MeV}$. At that threshold the light output is zero photons, with light output given by

$N=5001-\left(\frac{1}{\eta^{2} \beta^{2}}\right) \frac{\text { photons }}{\mathrm{cm}}(\mathrm{vis}$ (b) $1 \mathrm{e})$ ? $^{9}$

More important is the fact that the light is collected at the ends of the counter and so must be emitted at a Cherenkov emission angle large enough to allow total internal reflection. Figure 13 shows the operating region for the various particle types for an 

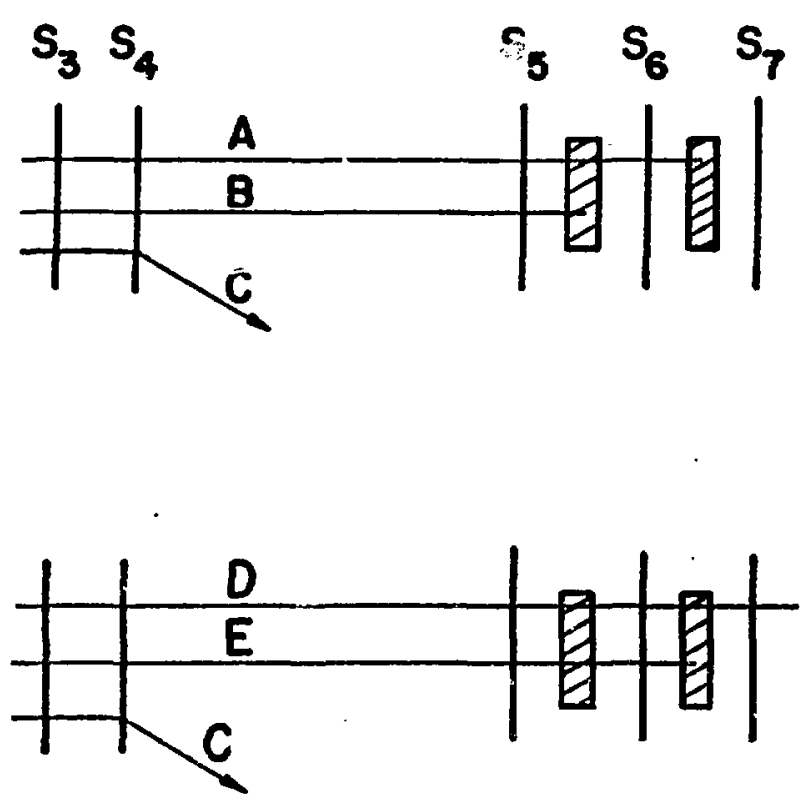

Fig. 12.

Trigger choices available when using the electronics of Fig. 3 and an additional scintillator and two absorbers.

n $=1.49$ Cherenkov counter. The $\pi, \mu$, and e particles contribute measurable signals for the $C_{L}$ counter. Other particles are detected principally by highenergy knock-on electrons. In addition to the possibility of detecting these particles with some low efficiency, the small Cherenkov light outputs for the $\tau, \mu$, and e particles also result in detection inefficiencies, necessitating investigation for eact. case (see Appendix G).

The second Cherenkov counter, $C_{D}$, is made from 5-cm-thick NE 425 Cherenkov material ( $n=1.49$ ) which, unlike the UVT Lucite, is doped with a wavelength shifter toward the more sensitive region of commercially available bi-alkali PM tubes $(\alpha=$ $425 \mathrm{~nm}$ ). The doping may also improve light transmission. More important, however, the reaulting light emission is isotropic more in the manner of a scintillation counter than the directional UVT Lucite counter. Because the initial light is still produced by the Cherenkov mechanism, its intensity has the same threshold and same $\beta$ dependence, but the light output is bette $\bar{c}$ ihan from equivalent thicknesses of UVT Lucite. Again, because of photoelectron fluctuations, the detection efficiency must be investigated for each use.

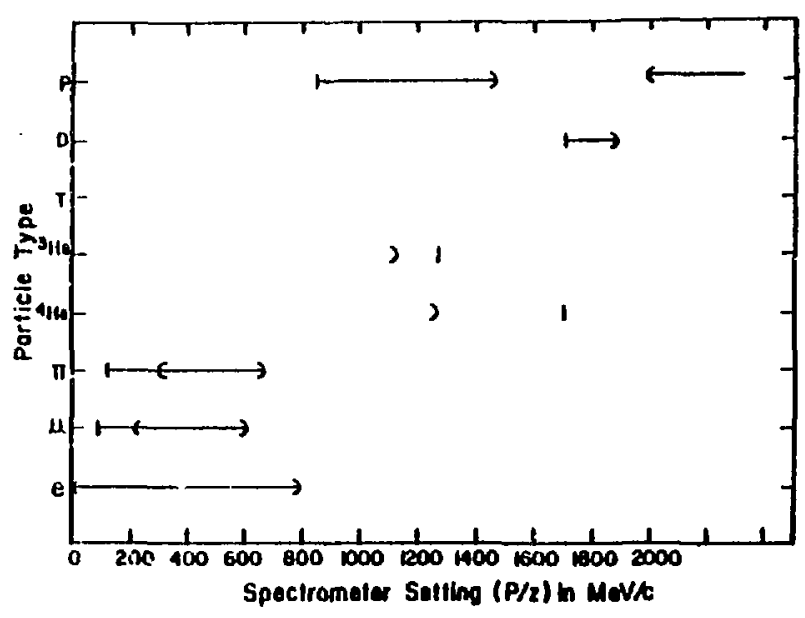

Fig. 13.

Regions of $n=1.49$ Cherenkov counter operation for different particle types as a function of magnetic rigidity. Start of the Cherenkov region is indicated by a vertical mark; the start of internal reflection is given by a left parenthesis; and the kinematic limit for production of a particle type by an $800-\mathrm{MeV}$ proton is given by a right parenthesis.

A Lit it: counter can operate between right and left parentheses while a NE 425 Cherenkov material should extend further down toward the vertical mark.

The rossibility of event selection with and without these Cherenkov counters allows on-line investigation of Cherenkov-counter performance. Even if they are not used in the trigger their presence is still latched in the CAMAC for off-line analysis.

5. Additional Hardware Possibilities. If a fully depleted, position-sensitive, solid-state detector or its equivalent were added following the target, more resolution would be provided on the scattering angle. Another possible addition is a gas Cherenkov $\left(C_{G}\right)$ counter to recognize electrons. The electronics for $\mathrm{C}_{G}$ are in Fig. 3, and other uses for the electronics already have been mentioned. Soon we intend to incorporate another device, "Maxwells Demon," which essentiaily interrogates all particles that pass by (except, of course, tachyons). 
6. External Particle Separation via Fast Electronics. No single method can distinguish all possible particle types and conditions of interest. However, in instances where a single technique may suffice, a means of independent verification is always desirable. A "truth" table of the following form is useful in designing an experiment within this framework.

EXPERIMENT FOR 1260-MeV/e 'He

\begin{tabular}{|c|c|c|c|c|c|}
\hline artiale & $\underset{\text { Time? }}{\text { In }}$ & $\begin{array}{c}\text { Stop in } \\
\text { Degrador? }\end{array}$ & $\underset{\text { Sirnal? }}{C_{2}}$ & $\begin{array}{c}\mathrm{C}_{\mathrm{D}} \\
\text { Sifnal? }\end{array}$ & $\begin{array}{l}\text { Pulse } \\
\text { Sine? }\end{array}$ \\
\hline${ }^{4} \mathrm{He}$ & yes & yes & no & no & $v_{a}$ \\
\hline "He & . & . not ki & tically & & \\
\hline $\mathbf{T}$ & no & yes & no & no & \\
\hline D & yes & no & no & no & \\
\hline $\mathbf{P}$ & Do & no & no & yes & \\
\hline
\end{tabular}

In this case one could look only at alphas, but suppose we reduce the energy sufficiently to allow ${ }^{2} \mathrm{He}$.

To anticipate an argument made in Sec. V, consider the problem as one of selecting subspaces in a multidimensional space. One projection is shown in Fig. 14 for $p=1.13 \mathrm{GeV} / \mathrm{c}$. For this figure we used the recommended 10-ns minimum pulse width, the OP. configuration, and worst-case pulse sizes using $60 \%$ transmission from center and $60 \%$ of the proton's photoelectron yield from the earlier calculation. Imagine that $C_{L}$ and $C_{D}$ split the space into multiple sheets, with $\pi-\mu$-e on one sheet $\left(C_{L}=\right.$ yes, $C_{D}=$ yes $)$ and $p$ on another sheet $\left(C_{L}=\right.$ no, $C_{D}$ $=$ yes $)$, and D-T. ${ }^{8} \mathrm{He}-\alpha$ on a third sheet $\left(\mathrm{C}_{\mathrm{L}}=\right.$ no, $\mathrm{C}_{\mathrm{D}}=\mathrm{no}$ ). Figure 14 then shows that ${ }^{\circ} \mathrm{He}$ and ${ }^{4} \mathrm{He}$ still will not be resolved.

The insertion of absorber material can modify the corresponding truth table in one significant way which shows that the use of absorber can be independent of the use of pulse height to distinguish betiveen particle types. The SLTSCT program can generate plots such as Fig. 15, which shows the amount of lead required to stop $1 \%$ and $99 \%$ of each particle type at $1129.3 \mathrm{MeV} / \mathrm{c}$. In the case of Fig. 15, one might choose absorber to eliminate $T$ and $\alpha$ from the trigger of the ${ }^{\circ} \mathrm{He}$ experiment or vice versa.

With such a family of hardware and the various projections, the experimenter can design an optimum trigger system together with his own list of possible contaminations and losses. For instance, a possible system for a ${ }^{4} \mathrm{He}$ experiment involves use of the absorber, an extra scintillator, and the timing and pulse-size information available from the setup shown in Fig. 16. The principal trigger would be $\left(W \cdot S_{d} \cdot S_{s}\right) \cdot\left(S_{b} \cdot S_{\bullet} \cdot \bar{S}_{\imath}\right)$ with large threshold settings, together with the backgrounds:
(1)
$\left(W \cdot s_{3} \cdot s_{4}\right) \cdot\left(s_{5} \cdot s_{6} \cdot \bar{s}_{7}\right)$
(Lower thresholds)
$\left(J \cdot s_{3} \cdot s_{4}\right) \cdot\left(s_{5} \cdot s_{6} \cdot s_{7}\right)$

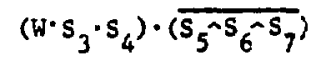
(4)

$$
\left(w \cdot s_{3} \cdot \bar{s}_{4}\right) \cdot\left(\overline{s_{5}-s_{6}-s_{7}}\right)
$$

This list can be extended and if proton backgrounds prove to be significant, the $N E 425$ counter could be added in veto.

Notice that for this example, the ${ }^{4} \mathrm{He}$ will lose part of its kinetic energy in the $S_{\mathbf{3}}, S_{4}$ pair. The actual flight time would then be longer than the Fig. 14 times and would fluctuate because of straggling. The SLTSCT program can provide a complete pictire of the spectrometer system and plots of the type shown in Fig. 14. In addition, the nuclear interactions included in the program (but not considered in Fig. 15) are available to provide estimates for the particle-los s corrections represented by items (2) through (4) above.

\section{B. Timing and Pulse-Size Information in the CAMAC System}

The hardware and electronics for the event trigger system are located at the detector area on the mainframe of the spectrometer. The discriminator thresholds and pulse widths, as well as the logic configurations for some of the coincidence units, can be remotely controlled from the $\mathrm{CCH}$. The timing delays indicated in Fig. 3 are also remotely contzollable.

Inside the $\mathrm{CCH}$, the timing and pulse-height signals read into the computer can be independently prepared for CAMAC in the way shown in Fig. 4. (This is one reason the LRS612 amplifiers were included in Fig. 3.) The equipment in the $\mathrm{CCH}$ is available for both monitoring and adjustment according to the experiment and need not duplicate the external setup shown in Fig. 3. The experimenter may set up his individual configurations from a 


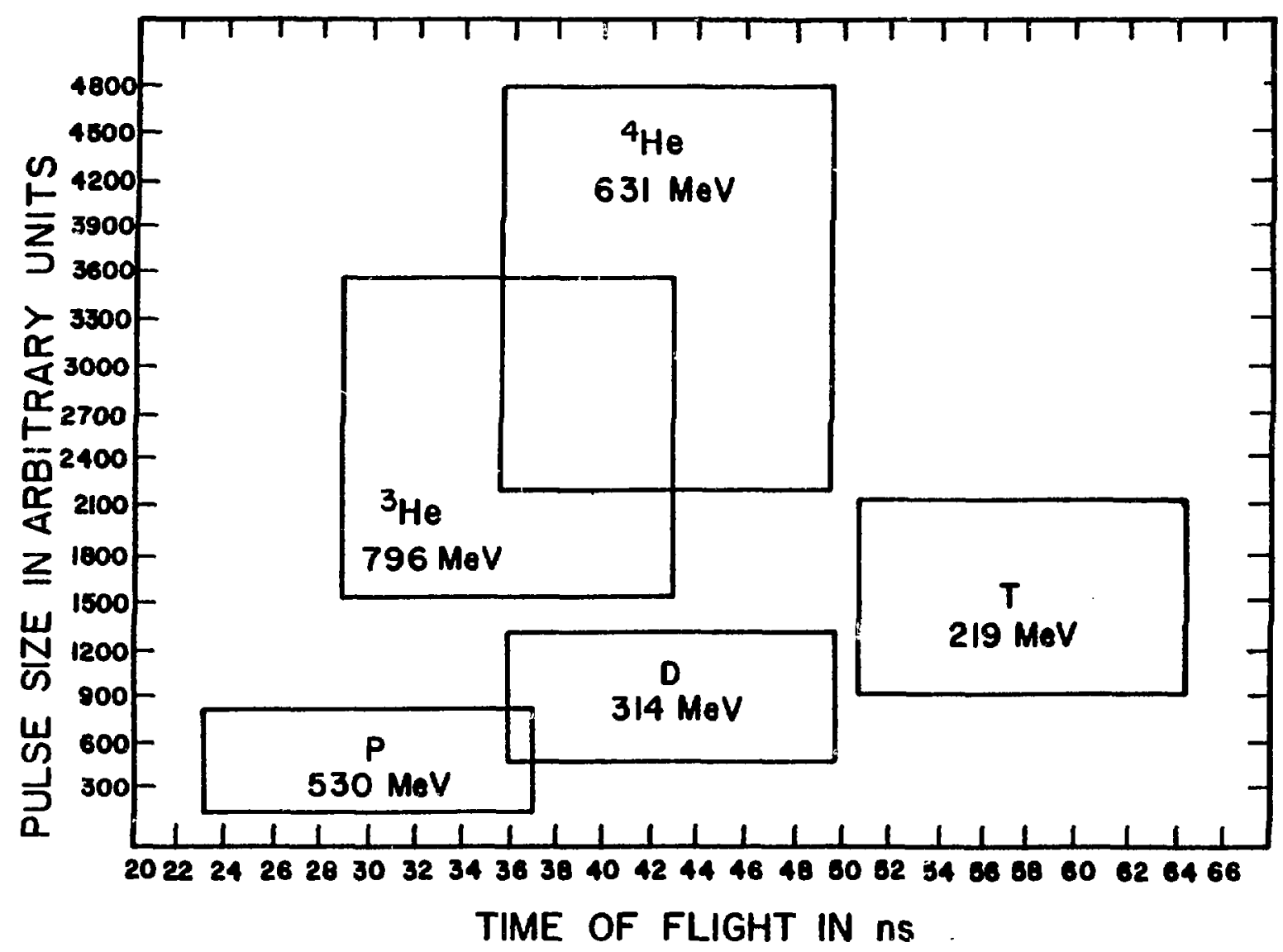

Fig. 14.

Two-dimensional plot of projections of pulse size and flight time for particles of $P / z=1129.3$ $\mathrm{MeV}$. These pulse sizes would be presented to the fast electronics, including straggling and scintillator attenuation not compensated for position. The times are those selected by the fast electronics coincidence and include the position variation and the pulse width.

selection of amplifiers, attenuators, and timing discriminators. Within the basic system, however, wire-chamber information, times for each scintillator's phototube, and pulse sizes for each phototube can be recorded. Also, a variety of scaler totals are provided, together with an additional list which can be interfaced if needed. Finally, an LRS 2340B strobed latch (Discriminator-CoincidenceBuffer) can record additional signal and logic information as a bit pattern for subsequent cross checks in the computer. Appendix A contains a list of recorded quantities and alternates. Most experimenters probably will accept the besic configurations in the $\mathrm{CCH}$ and concentrate on selecting dynamic ranges for ADC signals and fine-tuning amplifier system.s.
Possible additional electronics here would allow higher selectivity in the events for which data are accepted. Slow analog electronics could correct the fast timing by use of a phototube logic different from that already discussed. ${ }^{12}$ The CAMAC system and the detector system record the event information on the strobe signal from the fast electronics. The ADC, time-to-digital converter (TDC), and chamber hardware have "fast clear" or "abort" inputs to permit the rejection of data or termination of input without significant loss of time should the "slow" electronics say to do so. These electronics could be located with the CAMAC equipment in the $\mathrm{CCH}$ but their specific character has not been specified. Some specifications for one possibility 


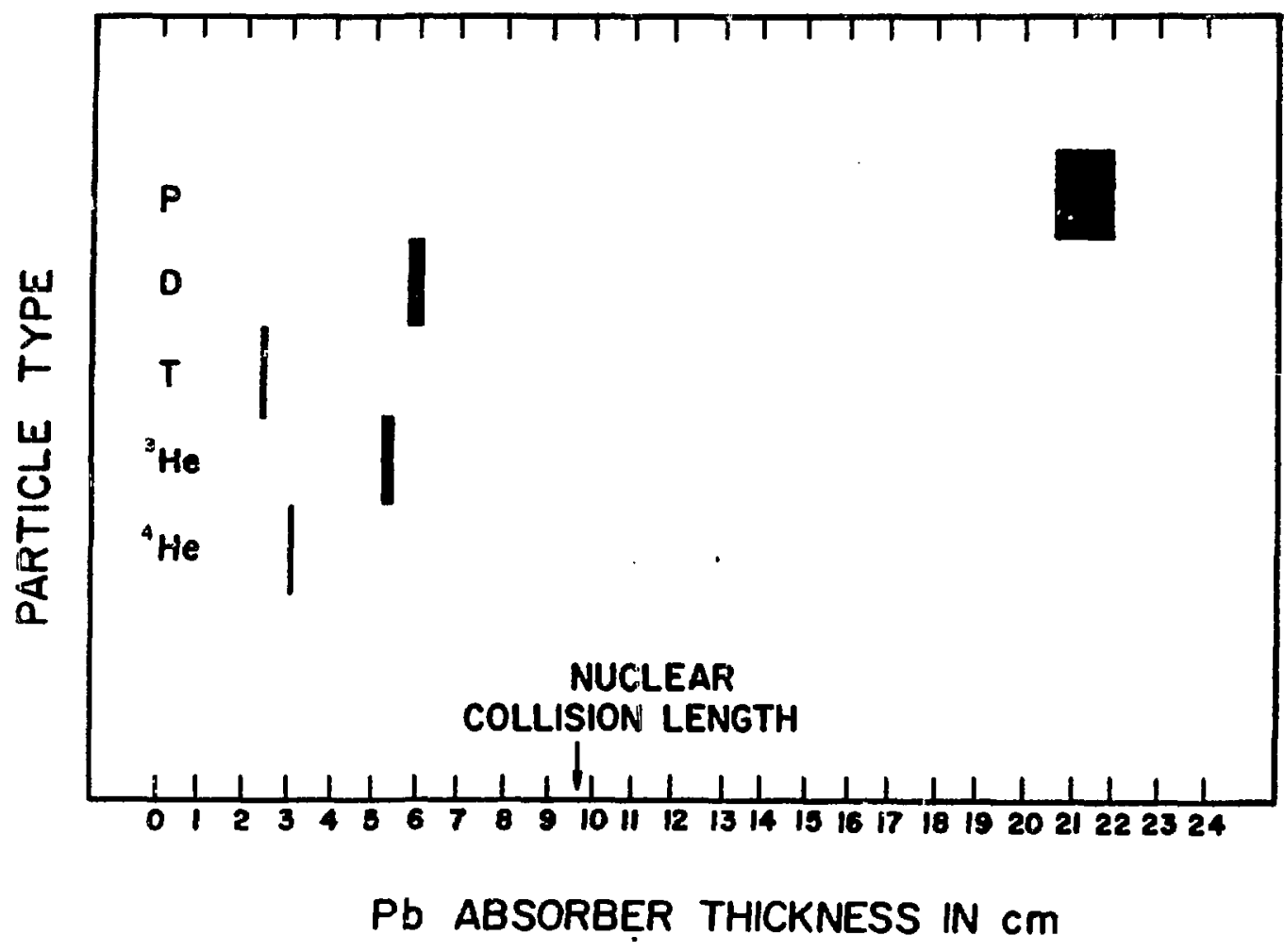

Fig. 15.

Range in lead for particles of $P / z=1129.3 \mathrm{MeV} / \mathrm{c}$, showing amounts of lead for $99 \%$ transmission and $1 \%$ transmission with nuclear processes ignored.

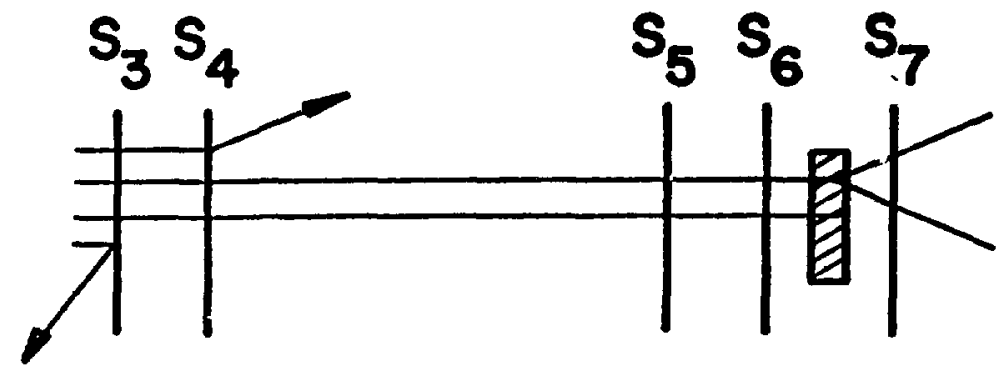

Fig. 16.

Possible setup for $(p, \alpha)$ experiments using the electronics of Fig. 3 and the hardware of Fig. 1.

which is consistent with the overall approach adop $i$ ted here are described in Appendix $\mathrm{E}$.

Also included in the basic system, located in the $\mathrm{CCH}$, is a pulser system for light-emitting diode (LED) devices" on each scintillator. They can be used for testing and alignment with the beam off, which should minimize setup times and permit more effective use of the beam time. The pulser system can also be arranged to trigger from the computer for on-line testing and monitoring. The computer would require separate calibration tables, but the CAMAC system and its contents, as well as the scintillators and fast electronics setup, could be tested. 


\section{Experiment Operation and Monitoring}

One way to monitor equipment during an experiment is to duplicate the measurements by another method. The system shown in Fig. 3 has advantages in this regard-among them the ability to continue an experiment when equipment fails. When properly used, it also gives one an added measure of confidence about the quality of the data. The major weakness of the system is not in realizing that something has gone out of tolerance but in determining where the fault has occurred. However, a properly interfaced system of LEDs helps considerably.

In addition to helping to assure the validity of the acquired data, the electronics provide two coincidences between the experiment's strobe pulse (which is itself timed by the $S_{3}$ leading edge) and $S_{3}$. Both coincidences have $S_{\mathbf{a}}$ timed to miss the strobe pulse for the $S$, pulse which initiated the strobe. In this way, "early" and "late" signals can indicate the presence of another particle in the counters at about the same time which may invalidate the timing and pulse-size data, or the signals can indicate simply that the electronics are busy.

The electronics design in Fig. 3 includes five possible interrupts. From the basic $S_{3}-S_{4}-S_{i}-S_{6}-C_{L}-$ $C_{D}$ system these interrupts can be generated from five logic configurations, each of which has the choice of its own coincidence timing. The basic trigger design consists of a coincidence (or veto) between the "front" counters $S_{9} \cdot S_{4}$, with the inclusion of any upstream counters or wire-chamber signals and the "rear" counters $S_{B}, S_{A}, C_{L}, C_{D}$. In the case of interrupts $1,2,4$, and 5 the rear signals can th formed from $\left(S_{b} \cdot S_{b}\right) \cdot\left(\right.$ any combination of $C_{L}, \bar{C}_{L}, C_{D}$, $\overline{\mathrm{C}}_{\mathrm{D}}$ ). The output pulse widths and the timing for coincidence with the front counters can be chosen differently for each of the four coincidences. Diagrams of the possibilities for interrupts 1,2,4, and 5 are shown in Fig. 17. With them and interrupt 3 a user can investigat $\epsilon$ most timing and scattering processes that lead to event losses or backgrounds.

Each interrupt type has on-line computer control. The possible interrupt coincidences are counted in scalers, but the interrupt is generated only if the coincidence itself is enabled by the computer. Thus the computer can select a sample of events from the different interrupt typos as specified by the user.
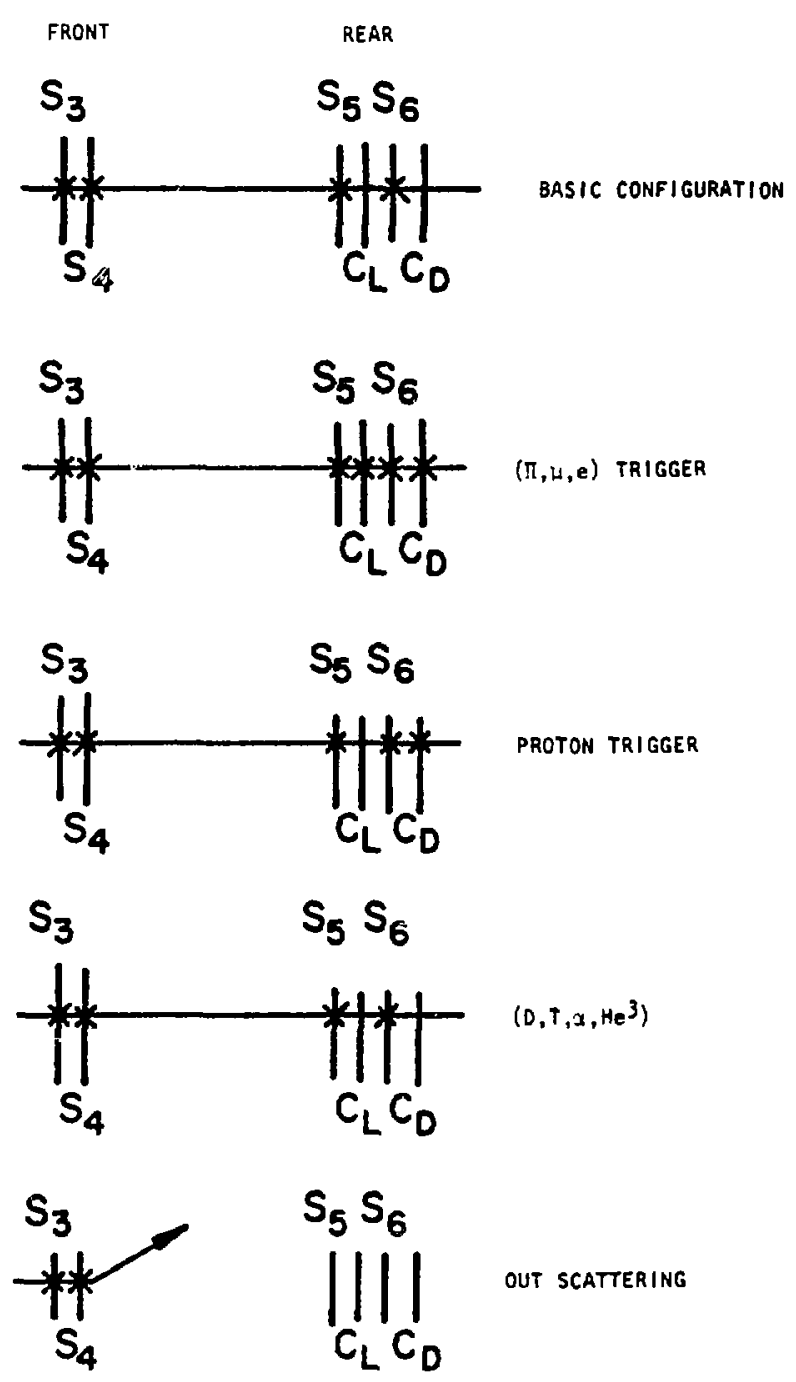

Fig. 17.

Schematic of particle paths and basic interrupts available with the hardware of Fig. 1 and the electronics of Fig. 3. Asterisks indicate counter output, i.e., a top/bottom coincidence or at least an output from the top or bottom phototubes.

However, all interrupts can be simultaneously enabled and recorded if desireci.

The user also can control the spectrometer aperture with the remotely movable $S_{\mathbf{8 , 4}}$ and $J$ counters which are already present in the coincidence units and need only be enabled locally or vis the computer. Considerable care must be exercised here 
because the various cross-section normalization/calibration data are involved.

\section{Cross-Section Normalization and Correction} Information

The last function of the overall system is to provide the information necessary to obtain normaiized and corrected cross sections from the data. For the normalization, the HRS has at least four devices:

(1) Beam current monitors (toroids),

(2) Secondary emission monitors (SEMs),

(3) Ion chambers (ISICs and ICs), and

(4) Target current monitors (scintillator telescopes).

To obtain a cross section, one needs to know the beam current with redundancy over a large range $(10 \mathrm{pA}$ to $10 \mu \mathrm{A})$ as well as a record of computer deadtime and detector inefficiencies.

To prevent unserviceable interrupts, destroyed data, or general confusion during the CAMAC readout, many people instruct the computer to turn off the NIM system. That option will be available at HRS through the interrupt module and the LAMPF gate generator. A more attractive possibility is to keep the NIM electronics working and provide scalers for the NIM electronics as well as for the beem monitors. Some of the scalers count all of the time, whereas others are inhibited whenever the computer is transferring data. This provides additional redundancy with simplicity in that there are no questions of crate on/off transition times. Naturally, when the scalers are inhibited, the NIM interrupt signals are also inhibited (see Appendix C).

\section{COUNTER CONSTRUCTION AND TESTING}

The scintillation counters $S_{2}, S_{6}, S_{6}, S_{6}$, and the Cherenkov counters $C_{\mathfrak{l}}, C_{D}$ are fabricated by Nuclear Enterprises of San Carlos, CA. The counter sizes shown in Table II were determined by simulating the spectrometer with the code SLTSCT̃.
TABLE II

DESCRIPTION OF SOME OF THE COUNTERS USED IN THE HRS

\begin{tabular}{|c|c|c|c|}
\hline Counter & Size $(\mathrm{mm})$ & Phototubes & Materinl \\
\hline $\mathbf{S}_{\mathbf{a}}$ & $140 \times 9.5 \times 1067$ & 2-EM19813B & NEi 10 \\
\hline $\mathbf{S}_{4}$ & $140 \times 9.5 \times 1067$ & 2-EM19813E & NE110 \\
\hline S, & $356 \times 12.7 \times 1829$ & 2-AMPEREX58DVP & NE110 \\
\hline S, & $356 \times 12.7 \times 1829$ & 2-AMPEREX58DVP & NE110 \\
\hline$C_{L}$ & $356 \times 25.4 \times 1829$ & 2.AMPEREX5BDVP & UVT Lucite \\
\hline $\mathbf{C}_{\mathrm{D}}$ & $356 \times 50.8 \times 1829$ & 2-AMPEREX58DVP & NE425 \\
\hline
\end{tabular}

Each counter has light pipes of twisted strips of UVT Lucite at each end of the long dimension. The light pipes end in tapered, conical adaptors, upon which a ring with internal conical taper is epoxied to provide the mounting support. Each counter is mounted in an aluminum frame supported by the rings at either end. The counter frame also serves as a gluing jig for the attachment of the phototubes with NE530 optical epoxy. Scintillation counters $S_{5}$ and $S_{6}$ also have 51-mm-long cylinders of polished UVT Lucite epoxied between the light-pipe end and the phototube to allow additional space for magnetic shielding and to insure that the shielding will extend at least half a photocathode diameter beyond the tube face.

The active areas of the counters are wrapped with a single layer of $0.175-\mathrm{mm}$-thick aluminum foil and a layer of black polyvinyl chloride (PVC) tape. The light-pipe regions have an additional layer of heavy black construction paper between the foil and the tape (to support the tape) and at least two layers of tape. Each photomultiplier is wrapped with PVC tape before installation and a Mylar collar separates the photomultiplier body from contact with the aluminum foil used to wrap the counter and light pipe. Also, the Amperex tubes have at leust one layer of 0.254-mm-thick Mylar outside the PVC tape for additional insulation. Each tube is also wrapped with mu-metal foil in addition to the usual mu-metal magnetic shield. Finally, the mounting 
rings on the light pipes are supported by iron tubes which slip over the phototubes.

The EMI phototubes are fitted with the LASL standard base, which is not adjustable, and the Amperex phototubes on $S_{6}$ and $S_{6}$ are fitted with Ortec 269 base assemblies. The $C_{L}$ and $C_{D}$ phototubes are fitted with similar bases obtained from Amperex. The 58DVP bases have provisions for adjusting the voltages used for accelerating and focusing the photoelectrons onto the first dynode. The objectives of the adjustment process are to provide, in order of importance:

(1) Uniform gain over the photocathode face, and

(2) Maximum gain.

The decision to adjust for uniform gain is based on the desire to minimize photoelectron fluctuations by collecting from the entire photocathode, making the match between effective photocathode area and counter area as good as possible. This decision implicitly favors the eventual $\mathrm{dE} / \mathrm{dx}$ identification over time resolution because it enhances the variation of photoelectron arrival time at the first dynode which depends on the collection path from the photocathode.

The adjustment involves the use of two oscilloscopes. The screen of one is placed in a lighttight box with the phototube and a lens to focus the oscilloscope trace onto the photomultiplier. The two oscilloscopes provide external "sawtooth" sweep signals and by interconnecting sawtooths and inputs, identical "raster" patterns are produced on the two oscilloscope screens. The photomultiplier signal is added to the input of the external oscilloscope 80 that the photomultiplier output is superimposed on the raster. If the light output of the oscilloscope inside the box is kept below the saturation level of the phototube and base, the picture on the second oscilloscope shows a two-dimensional picture of the combined efficiency of the photocathode and collection systems. Photographs of the oscilloscope output are shown in Fig. 18 for the phototube bases as delivered and after adjustment.

Light pulsers ${ }^{13}$ are placed at the center of the active regions and near each end. After wrapping and testing, a curious effect appears: the risetime from the light pulser on the scintillator is about $6 \mathrm{~ns}$ longer than that for the baro light pulsers during their tests. We believe the difference arises from the light-collection geometry.
Plateau curves and pulse-size spectra for localized cosmic rays can determine the operating voltage regions of the tubes. The operating witages are chosen for equal gains with the $1-\mathrm{MeV}$ conversion electron of ${ }^{207} \mathrm{Bi}$. The choice of yoltage is later verified with particles from the spectrometer by raising and lowering photomultiplier voltages to search for efficiency changes.

\section{COMPUTER INTERACTION WITH FAST ELECTRONICS}

The computer is expected to supply a variety of signals and fulfill the following functions:

(1) Start/stop experiment at user's command either via push-button or keyboard command or after a preset number of microcoulombs (see Appen$\operatorname{dix} \mathrm{C}$ ),

(2) Provide a way to record the deadtime from the data-taking operations (see Appendix C),

(3) Use beam time efficiently, taking nonevent information between beam gates on a priority basis,

(4) Select the logic configurations for the electronics:

(a) Before the rur, by enabling interrupt types in the MBD and CAMAC interrupt module, and

(b) On-line, through use of the CAMAC output gate to specify the logic in the LRS 365 ALP modules,

(5) Sample different event types as specified above by using the output gate to enable the appropriate interrupt types (see Appendix C), and

(6) Use the various constraints on the input data to monitor the function of the hardware.

In previous sections we presented a system of fast electronics with emphasis on selectivity. At the same tinse, problems of event losses and backgrounds were constantly mentioned and provisions were made for investigating them (see Appendix D). Depending o: the individual experiment and its event rate, the most desirable trigger definition is the acceptance by the fast electronics of a large number of events with low selectivity. The computer can then perform a more sophisticated, efficient data-rejection analysis with fewer biases. The computer has three major advantages in this area. 

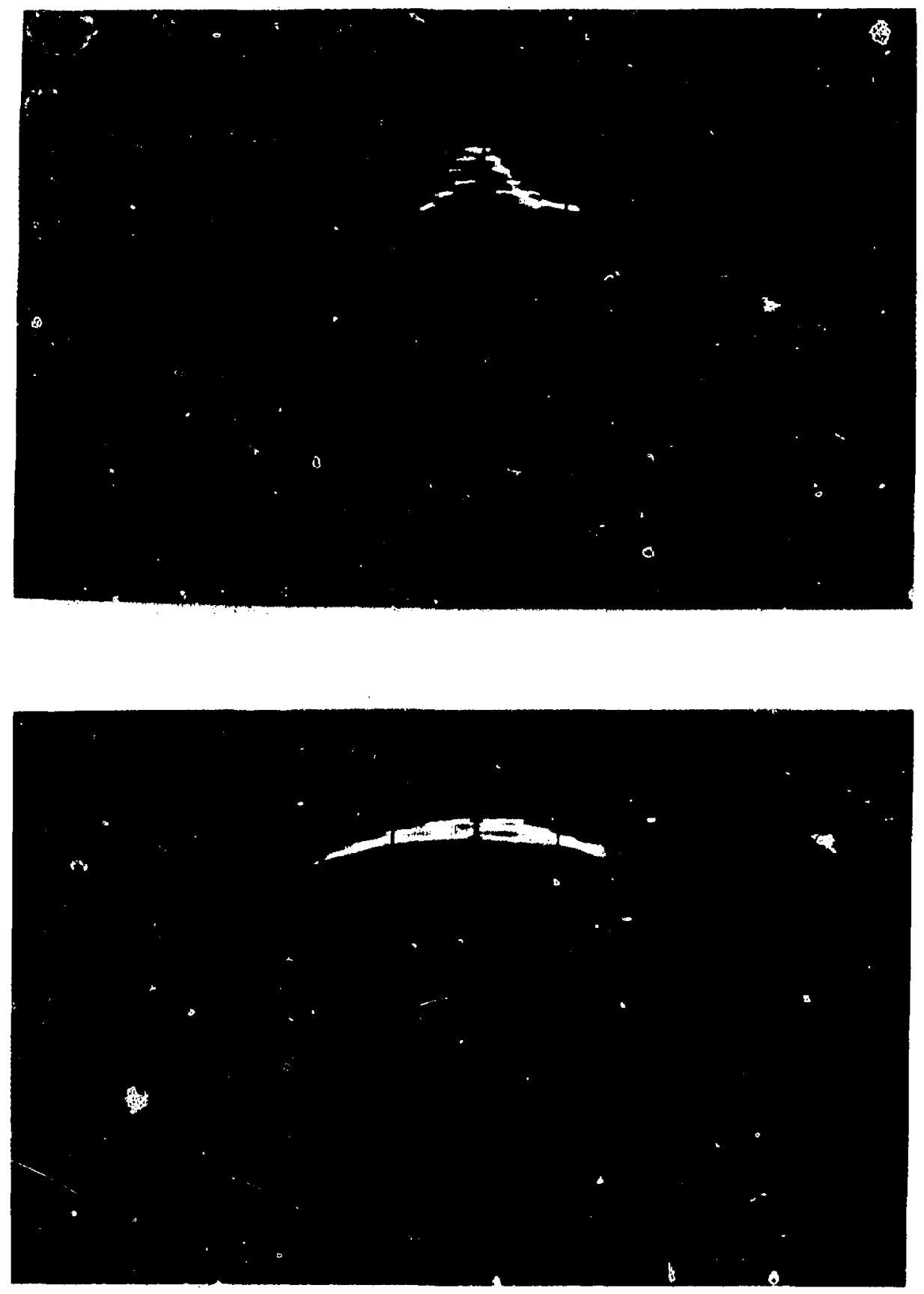

Fig. 18.

Oscillographs of phototube base adjustment. Above, base output as delivered; below, typical output after adjustments. Each display is a plot of phototube output as a function of location on the tube face. 
First, there is what we call sensitivity, which is based on the ability to make certain simple corrections to the data in the computer. Consider the example of Fig. 14, which is a projection of the fast electronics data onto the time and pulse-size plane using the recommended setup for the trigger elec. tronics. In this projection, the regions for some particles overlap, indicating that particle separation in those subspaces requires additional hardware constraints such as the Cherenkov counters if the fast electronice are going to provide $100 \%$ separation. For comparison, Fig. 19 shows the predicted subspaces afier correction in the computer on the basis of the following differences:

(1) Variables of the type put in the computer are used, and

(2) Location effects are removed using wirechamber data.

Clearly, separation with the computer is better because there is greater separation between the gurfaces of the hypercubes occupied by each particle type. This situation always occurs to some extent as can be seen by considering the fast electronics as defining a multidimensional space made up of a series of distribution functions, $f\left(x_{1}\right)$, which the various trigger conditions combine into forms of the

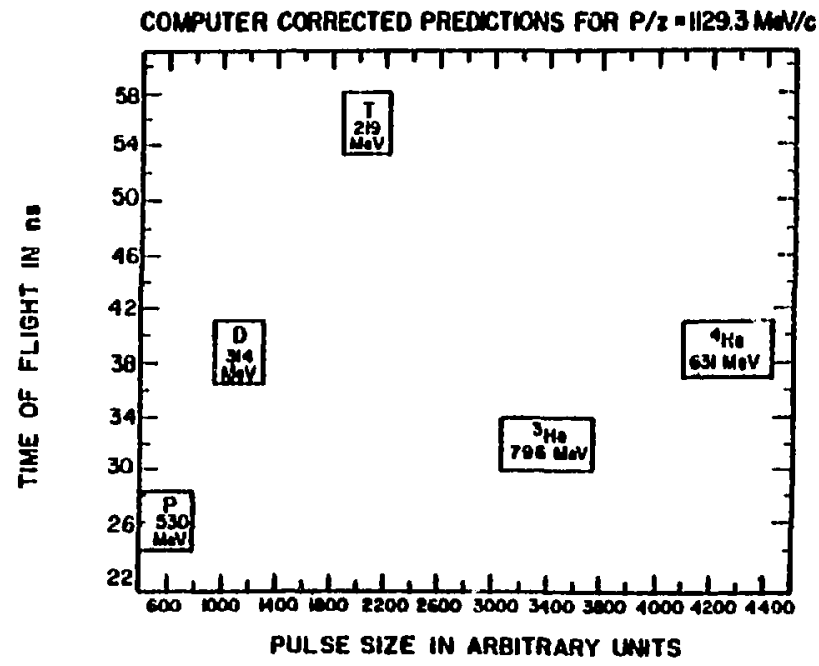

Fig. 19.

Pulse size us flight time, showing regions predicted for each particle type using information available in the computer analysis of the events. The pulse-height information for all particle types is fully resolved. type $f\left(x_{1}\right) f\left(x_{2}\right) f\left(x_{2}\right) \ldots$ In short, the "result function" is then the outcome of a sequence of cuts on the individual $f\left(x_{1}\right)$ which comprehend the various instrumental and statistical uncertainties likely to arise. As a result, windows large enough to minimize any significant bias must be used. The trigger system shown in Fig. 3 was therefore intended to be used with comparatively loose timing, with primary emphasis on the anode-timing setup.

The second advantage of using the computer is its potentially more sophisticated logic. For instance, the "result function" determined with the fast electronics does not consider that the set $\left(x_{1}\right)$, the data point in the hyperspace, is highly redundant and not independent. In contrast, the computer can convert the raw data space into one of lesser dimension and independent subspaces. Furthermore, this can be done in a variety of ways, independently of the electronics of Fig. 3. Although some of the simpler alternatives could be implemented in the fast elertronics, ${ }^{11,12}$ it would seldom be preferable except for very low cross section experiments. A CAMAC module which we believe would be very useful in such a case is described in Appendix F.

A third advantage of the computer is accuracy. For example, the subspaces of location-corrected particle times can have a series of "metric" spaces imposed on them, functions $f, g$, $h$, etc., measuring the distance to the data point from the "ideal proton," "ideal alpha," and the like. Each of these functions will have a known probability distribution associated with each variable's distance and one can combine the resulting probabilities, allowing for the redundancy in each subspace. Then the redundancy car improve the resolution as discussed above. In addition, from each of the particle characteristics and their associated probabilities, the confidence level for identification of each particle type is available. This reduces instrumental background in conventional cross-section measurements and also considerably improves the sensitivity of the system to rare events. It seeme to be a safer and more convenient method of identification because it requires only calculation instead of more arbitrary cutting and sorting and produces a single statement of particle types and confidence levels. The identifications can be used to indicate the efficiency of the selection process or might be used to linit the amount of tape-writing by rejecting obviously undesirable events. 
Many data will be required to "construct" the data acquisition system for a given experiment. The computer will have tables, such as Table III, which are listed according to the accelerator energy. Interpolation on this table will then be carried out for any particular spectrometer setting. Computer listings of the basic tables and calculations discussed in the report will be available for reference in the $\mathrm{CCH}$.

\section{RESULTS}

A number of techniques have been discussed, together with methods for combining them to arrive at a reasonably general particle-identification system. The system could be operated manually or be remotely controlled via CAMAC. Our purpose here is to show that the basic method is viable and functions essentially as predicted. For this to be the case, it is important for the individual counters to function predictably. Because of their sizes and dispositions in a new experimental area, it was not clear that this would be the case. In fact, we found that the singles count rates in the large counters $\left(S_{6,0}\right)$ were one of the most sensitive indications of misaligned or poorly tuned beam.

For the results shown below, the spectrometer was set at $\theta_{\mathrm{L}}=25^{\circ}$, where the coherent cross sections are rather small so that the signal-to-noise ratio would not be particularly good. A $170-\mathrm{mg} / \mathrm{cm}^{2}$ scintillator target was used with average beam currents of $\simeq \mathbf{2 0}$ to $40 \mathrm{nA}$. The spectrometer was set to look for elastic scattering $(\mathrm{P} / \mathrm{z}=1.43 \mathrm{GeV} / \mathrm{c})$ with $800-\mathrm{MeV}$ incident protons. Figure 20 shows some on-line dot plots of the TOF taken between $S_{\text {, and }} S_{\text {c versus the }}$ geometric means of the top and bottom pulse heights for one of the inner counters $\left(S_{4}\right)$, one outer counter $\left(S_{b}\right)$, and the average of the geometric means of two inner counters $\left(S_{3}\right.$ and $\left.S_{6}\right)$. All data were triggered by an $S_{8} \cdot S_{4} \cdot S_{8} \cdot S_{6}$ coincidence. There is clearly more noise in the pulse-height distribution of the larger counter $\left(S_{5}\right)$ and it is easy to worsen this significantly, but in this particular case there was no special effort to improve it very much. If one looks at just the average of $S_{\text {, and }} S_{4}$ in the lower figure, this background is essentially eliminated. Figure 21 shows the distribution of projected pulse heights for the counters in Fig. 20. Notice that the protons and deuterons are separated in pulse height for the $S_{8,4}$ average to better than 1 per 6000 . ' $\mathrm{Ti}$. time separation is even better. Using the minimum pulse height in $S_{2}$ or $S_{4}$ gives comparable results. Using the puise height in $S_{3}, S_{4}, S_{b}$, or $S_{0}$, after adjusting their relative gains at the bases or in the computer, provides significantly better results and also eliminates the background problem in the outer counters so long as the singles rates are reasonable $\left(\leqslant 10^{\circ} / \mathrm{s}\right)$.

This is not a particularly good example of the general method because the so-called hypercubes were already separated in the TOF coordinate. As a result, increasing the separation in another coordinate makes little difference until that separation becomes comparable to the first one. A better example is shown in Fig. 22, where the situation corresponds to that predicter in Fig. 19. To insure the possibility of seeing all of the heavier particles, the spectrometer was set at $P / z=1.08 \mathrm{GeV} / \mathrm{c}$. Everything else was left the same. Although the ratio of deuterons to protons $(D / p)$ has increased and we now see tritons fairly easily (roughly 1 for every $10^{4}$ protons), we still see no ${ }^{3} \mathrm{He}$ or ${ }^{4} \mathrm{He}$. Of more importance is the increased variation in the TOF of all of the particles, the improved pulseheight separation between $p$ and $D$, and the virtual absence of data anywhere in the plot that cannot be associated with a real event of potential interest.

The increased variation in TOF comes from the fairly uniform use of the full counter area as opposed to that used by the more localized elastic and lowlying inelastic spectrum used above. The increased spread leads to a worse TOF separation even though the separation between centroids has increased. On the other hand, the decreased momentum ir.sreases the energy loss and therefore maintains the pulseheight separation. In this case, the particles are separated as well by pulse height as by TOF.

So far we have concentrated on the heavy particles ( $p, D, T$, etc.) and the techniques for separating them because they are the ones of interest to the greatest number of experiments. There are also approved experiments which involve mesons and require the use of the Cherenkov counters. The $(p, \pi)$ reaction to discrete final nuclear states is discussed in Appendix G with specific reference to the HRS setup because it is an interesting example of how this system can be used for these kinds of experiments. 


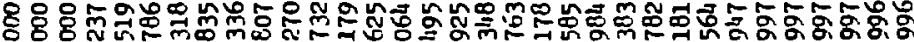
ن

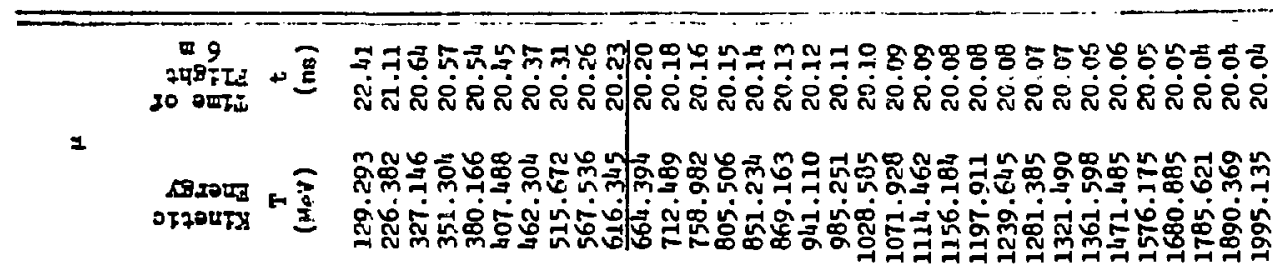

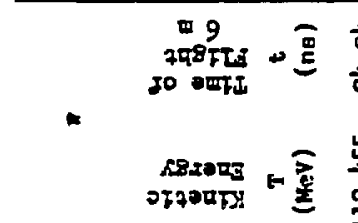

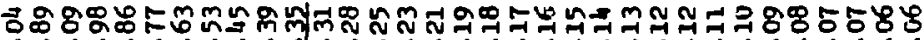
สี่ ส่ ฉิ่

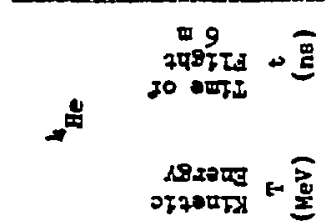

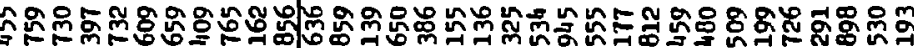
न

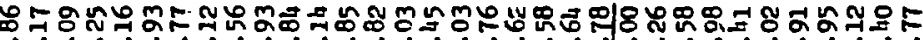

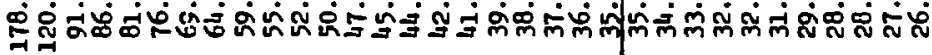

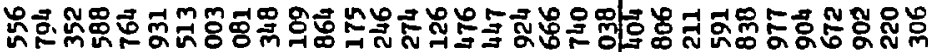
लิ่

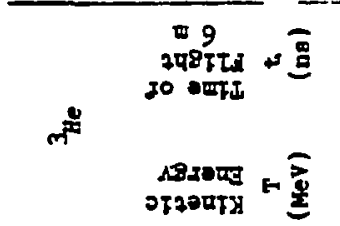

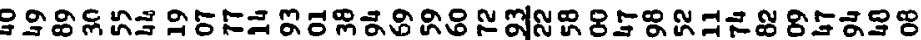

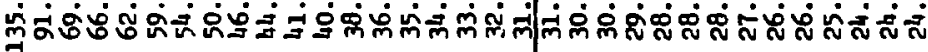

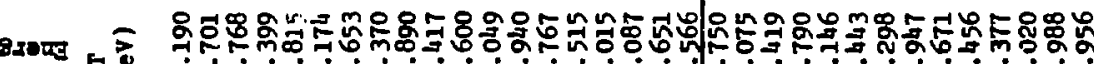
लिं

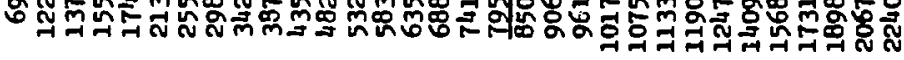

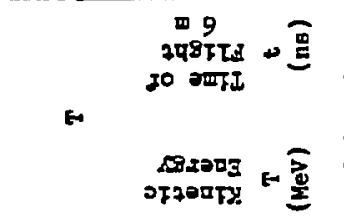

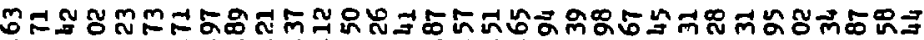

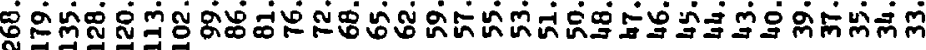

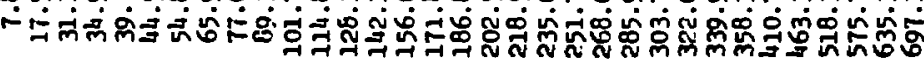

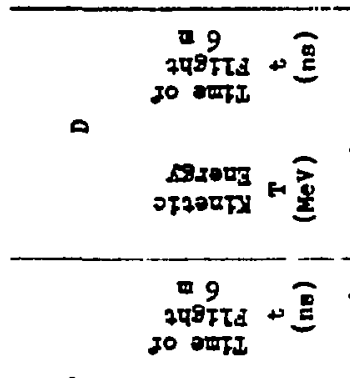

a.

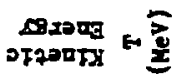

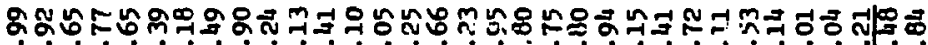

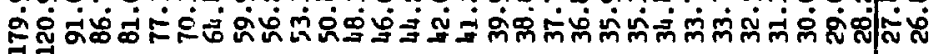

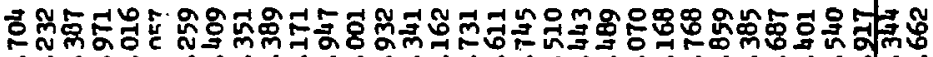
न

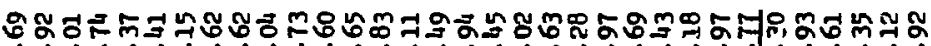

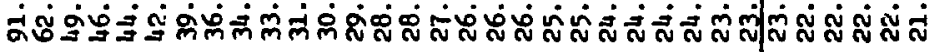

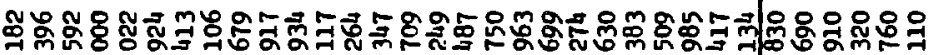
ஸ்

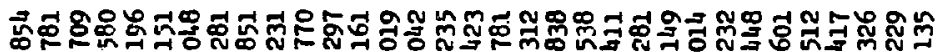
ฉें 


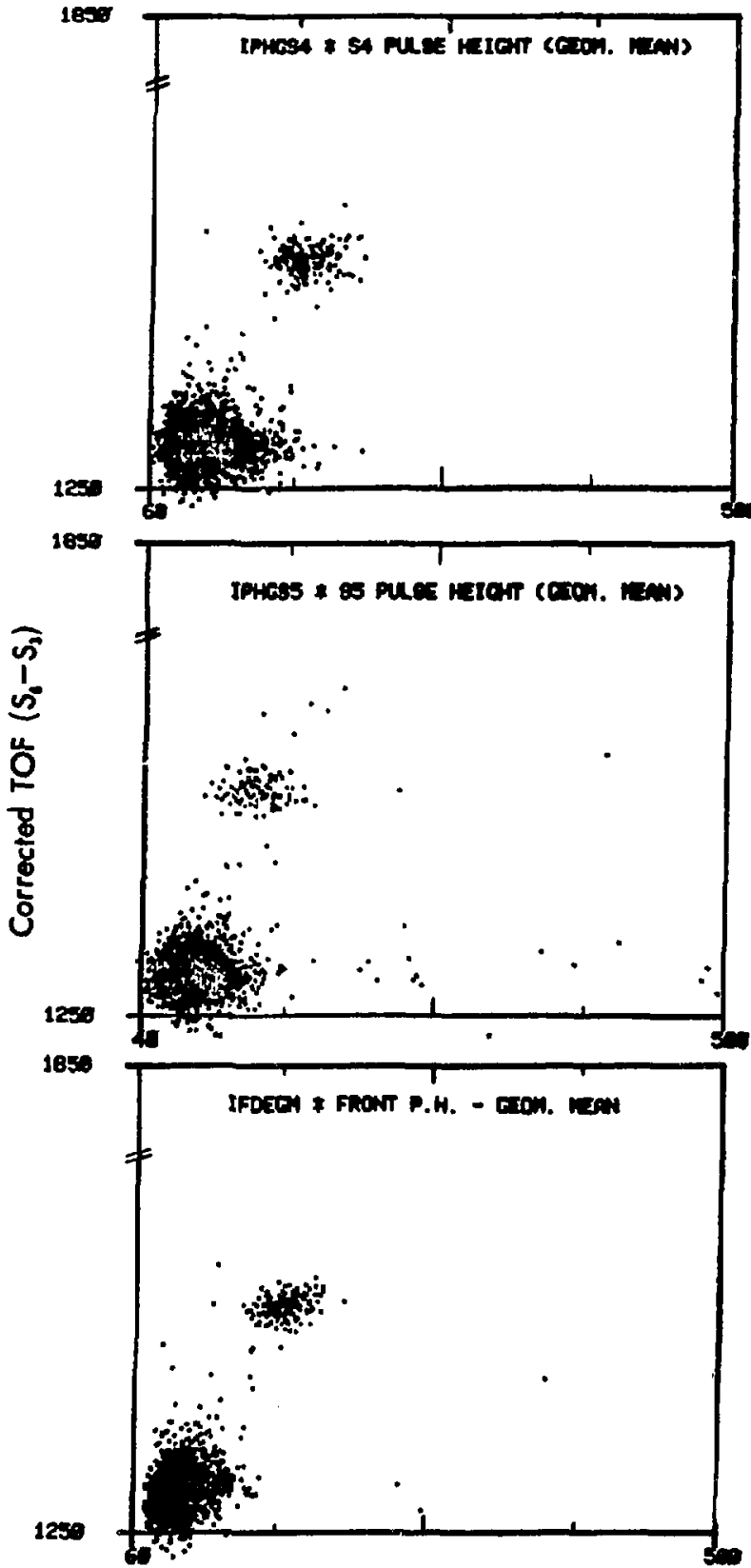

Pulse Height in ADC Channels

\section{Fig. 20.}

Typical on-line dot plots of ccinputercorrected TOF vs pulse height in units of $A D C$ channels for counters $S_{4} S_{8}$ and the average of the individual geometric means of $S_{3}$ and $S_{4}$. (Approximately $10^{s}$ proton events.)

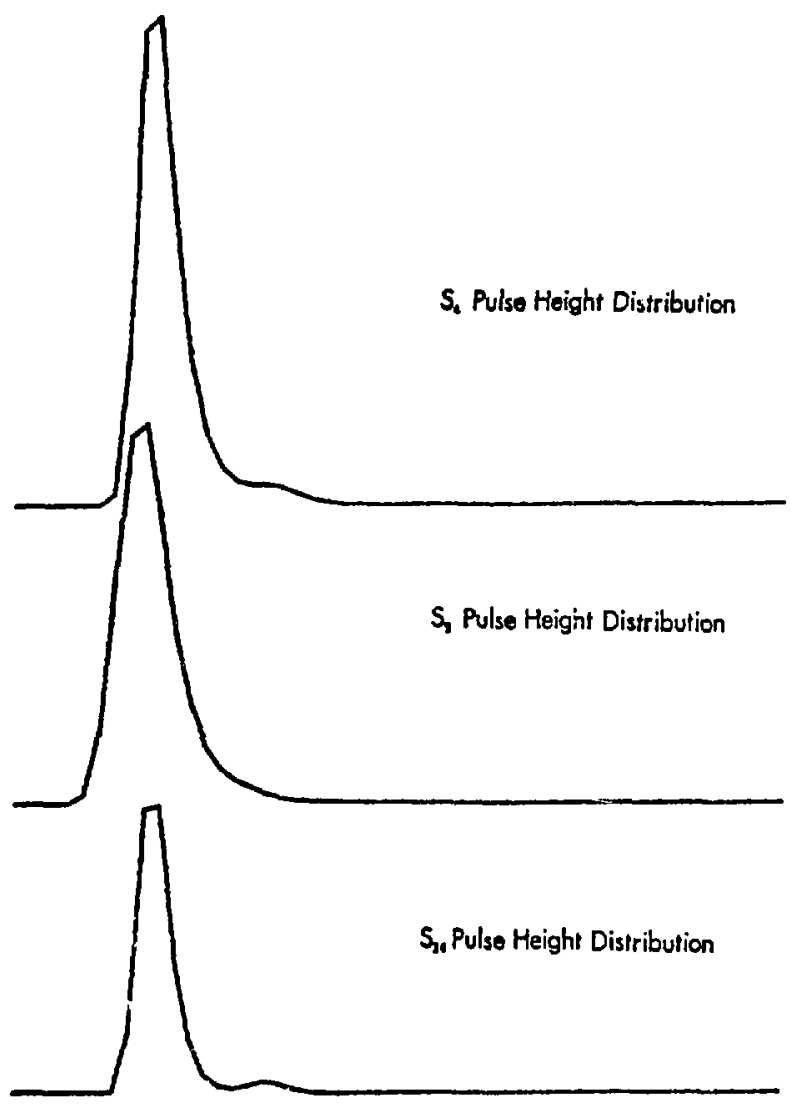

Fig. 21.

On-line projections on the pulse-height coordinate, showing number us pulse height for a number of $A D C$ channels similar to that used in Fig. 20. Vertical scale on the bottom plot is twice that of the other plots because of the better resolution obtained.

The main purpose of the system is to maximize the information content obtainable from a "typical" crose-section experiment. Because of the unique character of the facility, considerable effort has been put into the design simulation of the counter stack to insure its compatibility with the spectrometer optics and the various experiments. Use of the full momentum acceptance of the spectrometer and redundant identification of all particle types was given high priority. The results presented above, obtained with no shielding, are considered encouraging. 


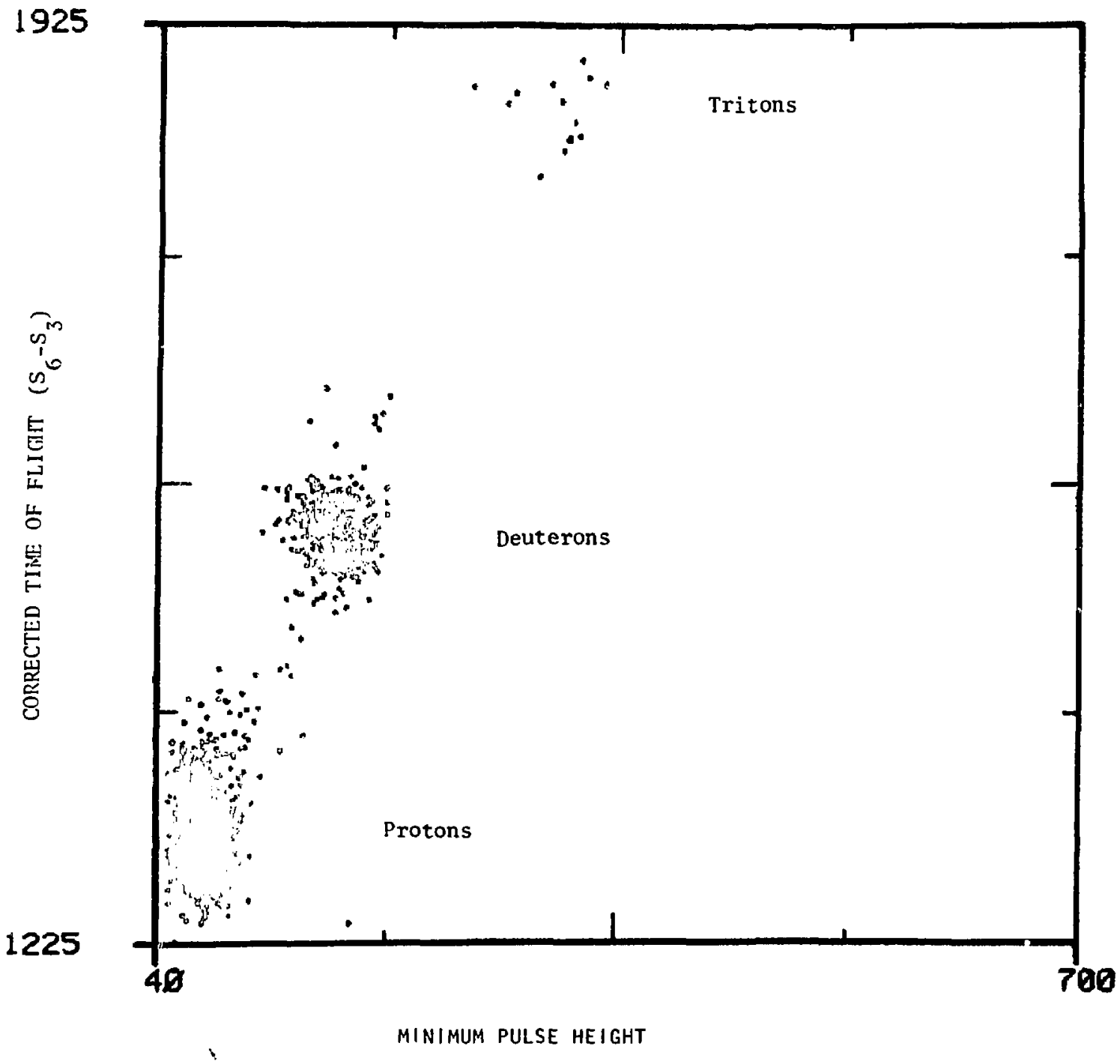

Fig. 22.

On-line results corresponding to the predictions of Fig. 19. The ordinate is the minimum of the geometric means of $S_{3}$ and $S_{4}$. The ratios of the heavier particles to the number of protons (primarily quasi-elastic) have increased but there is still no evidence of any ${ }^{3} \mathrm{He}$ or ${ }^{4} \mathrm{He}$. 


\section{COUNTING-HOUSE SIGNALS}

In this appendix is a list of signals provided to the $\mathrm{CCH}$ by the fast electronics of Fig. 3. These signals were intended to provide information about the selected events in CAMAC ADC and TDC modules as well as rate and normalization information in CAMAC scalers. They might also be used to provide the signals necessary for additional decision-making electronics.

\section{ADC INPUT}

The photomultiplier signals used in the fast electronics are not absorbed in the modules but ere made available for further use by the high- $\mathrm{Z}$ bridge features of the discriminators. These signals are then sent to the $\mathrm{CCH}$ where the following photomultiplier signals are available:

$S_{8} U$
$S_{8} D$
$S_{6} U$
$S_{6} D$
$S_{8} U$
$S_{6} U$
$S_{8} D$
$C_{L} U$
$C_{L} D$
$C_{D} U$
$C_{D} D$.

These photomultiplier signals are used for pulse-size measurements in a CAMAC ADC. Another possibility is to split the photomultiplier signals, as shown in Fig. 4, for use with a timing discriminator is provide signals to the CAMAC TDC for the measurement of particle flight times. Twenty-five RG/U 59 cables are instailed for high voltage, 25 RG/U 8 cables for fast, low-level signals such as those listed above, $80 \mathrm{RG} / \mathrm{U} 58$ cables for logic level signals, and more than 200 twisted pairs for control installed between the top level of the spectrometer frame and the $\mathrm{CCH}$.

\section{TDC INPUT}

Discriminator logic pulses from

$\mathrm{S}_{3} \mathrm{U}$

$\mathrm{S}, \mathrm{D}$

S.U

S,D

$S_{6} \mathrm{U}$

$\mathrm{S}_{6} \mathrm{D}$

$S_{0} U$

$\mathrm{S}_{6} \mathrm{D}$

$\mathrm{C}_{\mathrm{L}} \mathrm{U}$

$\mathrm{C}_{\mathrm{L}} \mathrm{D}$

$\mathrm{C}_{\mathrm{D}} \mathrm{U}$

$\mathrm{C}_{\mathrm{D}} \mathrm{D}$

are also available in the $\mathrm{CCH}$ and are used to provide signals to the CAMAC TDC for the measurement of particle flight times. They also provide the opportunity to monitor singles rates.

\section{SCALER INPUT}

In addition to the $\mathrm{CCH}$-generated signals, such as the pulse trains from beem-current monitors, the fast electronics of Fig. 3 supply logic signals for scalers from the points listed above as well as from

Interrupt 1, Coincidence

Interrupt 2, Coincidence

Interrupt 3, Coincidence

Interrupt 4, Coincidence

Interrupt 5, Coincidence

Strobes

S,

S.

S。

S.

$\mathrm{C}_{\mathrm{L}}$

$C_{D}$

$S_{9} \cdot S_{4}$

$\mathbf{S}_{8} \cdot \mathbf{S}_{6}$ 


\section{LATCH INFORMATION}

In addition to the scaler infcrmation, the following pulses appear in a CAMAC latch to provide easily accessible information about each event:

Interrupt 1

Interrupt 2
Interrupt 3

Interrupt 4

Interrupt 5

Early

Late

All of the signals available in items (I-IV) above, including the $J_{1,2}$ and $S_{1,2}$ signals.

\section{APPENDIX B}

\section{NONSTANDARD ELECTRONICS}

The NIM fast electronics, with the exception of the remotely programmable logic delay, ${ }^{3}$ are ccmmercially available LeCroy Research Systems modules or minor modifications thereof. A complete file of the descriptions and specifications of all the modular electronics (both NIM and CAMAC) has been deposited in the HRS counting house for reference. The following modules are mentioned here to assist the reader's understanding of the circuit diagram of Fig. 3.

1. LRS 621BLPZ. LRS 621 is a quad $100-\mathrm{MHz}$ updating discriminator with a threshold of -30 to $-1000 \mathrm{mV}$ and an output pulse width of 5.5 to 1000 ns. Threshold and width adjustments are made by potentiometers located at the front panel, but an additional 14-pin connector on the rear panel of the module allows the threshold and width of each channel to be controlled by an externally applied dc voltage. In addition, a high- $Z$ bridge option is factory-installed in the HRS units. The internal $50 \Omega$ termination is removed from the input and the input signal is routed back and out through the nearest output connector. This signal may be reused or terminated at the front panel with 50 . In either case it must be properly terminated with $50 \Omega$.

2. LRS 622. LRS 622 is a quad twofold coincidence module which uses much of the basic circuitry of the LRS 621 discriminator. The two 622 in- puts are summed and the 621 threshold control pot is replaced by a two-position switch to select thresholds corresponding to AND and OR functions. The manufacturer supplied information for local modification to allow remote control similar to the $621 \mathrm{BLP}$. The pulse width is adjusted by dc voltage applied to the rear panel connector; AND/OR is selected by $+5 / 0$.V TTL levels applied to the rearpanel connector. For remote operation the function selector switch must be in the OR position. Without the rear-panel connection the behavior is like the ordinary 622 . Voltage assignments for the rear connector are attached to the side panel of each module.

3. LRS 365ALP. LRS 365 is a dual fourfold voter coincidence module. Each channel has four inputs and a veto input. Each of the four inputs may be enabled or disabled by insertion of front-panel programming pins. Also selected by front-panel programming pins is the voter-coincidence level, from 1 to 4 . The output width of the 365 is selected by a front-panel potentiometer. The 365ALP uses a rear-panel connector to which dc voltages can be ap. plied to select inputs or coincidence levels. These dc voltages function in the same way as the front-panel programming pins, which must be absent for remotely controlled operation. Pulse widths are controlled only by the front-panel potentiometer. 
4. Remotely Programmable Logic Delay. This module contains 16 independent channels of delay for fast NIM logic pulses. It can be controlled at the delay unit itself (REMOTE), in the $\mathrm{CCH}$ from a CAMAC unit (LOCAL), or by computer. Each channel has a fixed delay of $20 \mathrm{~ns}$ and a variable delay from 0 to $101 \mathrm{~ns}$ in 1-ns steps with a maximum accumulated error of \pm 1.5 ns. Inputs require NIM logic pulses of $\leqslant 6-n s$ width into $50 \Omega$ internal termination to obtain pulse-width fidelity of better than \pm 1 ns between input and output for instantaneous rates of $\leqslant 50 \mathrm{MHz}$. Dual outputs (LEMO)

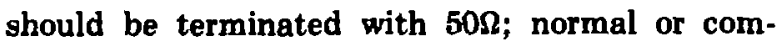
plementary output is selected by internal switches. Rise- and fall-times of output signals are faster than $1.3 \mathrm{~ns}$.

\section{APPENDIX C}

\section{CAMAC SCALER SYSTEM}

The beam monitors and NIM electronics run continuously, but the CAMAC scalers are gated in different ways. For some monitors, such as the types which report integrated charge, the scaler is gated on for the entire run because the charge integrator might complete an integration cycle outside the usual beam gate. In addition, the monitor and detector signals can be scaled with different gates to determine deadtime corrections and monitor counter backgrounds between beam pulses. The following scaler gate/inhibit signals are generated:

(1) RUN

(2) RUN - BEAM
(3) RUN - BEAM

(4) RUN - BEAM

These are made using the LAMPF Gate Generatoris which has the following facilities:

(1) RUN and RUN flip-flop with TTL output. The state is chosen by front-panel RUN and STOP buttons and front-panel TTL RUN and STOP inputs,

(2) TTL beam gate output,

(3) TTL-NIM, NIM-TTL, TTL-TTL converters,

(4) TTL coincidences, and

(5) Visible scaler and presettable counter with TTL signal output.

The CAMAC scaler arrangement is:

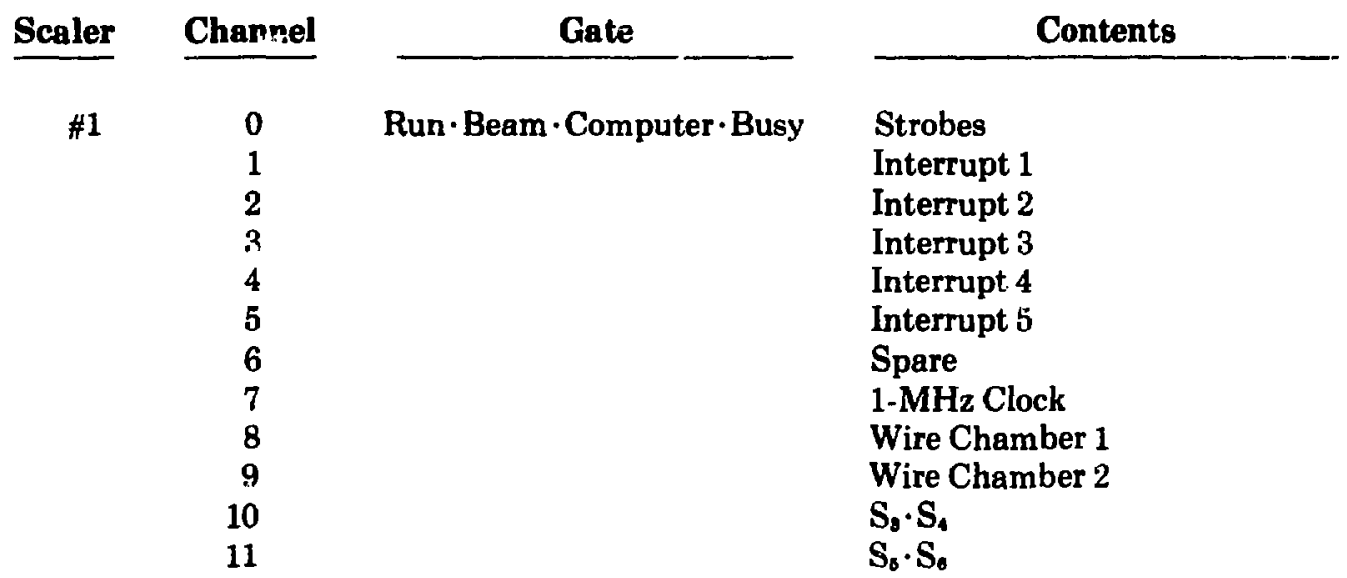




\begin{tabular}{|c|c|c|c|}
\hline Scaler & Channel & Gate & Contents \\
\hline \multirow[t]{12}{*}{$\# 2$} & 0 & \multirow[t]{12}{*}{ Run $\cdot$ Beam } & Strobes \\
\hline & 1 & & Interrupt 1 \\
\hline & 2 & & Interrupt 2 \\
\hline & 3 & & Interrupt 3 \\
\hline & 4 & & Interrupt 4 \\
\hline & 5 & & Interrupt 5 \\
\hline & 6 & & $\mathbf{S}_{\mathfrak{g}}$ \\
\hline & 7 & & S. \\
\hline & 8 & & $\mathbf{S}_{6}$ \\
\hline & 9 & & $\mathrm{~S}_{6}$ \\
\hline & 10 & & $\mathrm{~S}_{8} \cdot \mathrm{S}_{4}$ \\
\hline & 11 & & $\mathrm{~S}_{\mathrm{B}} \cdot \mathrm{S}_{8}$ \\
\hline \multirow[t]{12}{*}{$\# 3$} & 0 & \multirow[t]{12}{*}{ Run·Beam } & $\mathrm{S}_{8} \mathrm{U}$ Singles \\
\hline & 1 & & $\mathrm{~S}_{8} \mathrm{D}$ Singles \\
\hline & 2 & & S،U Singles \\
\hline & 3 & & $\mathrm{~S}_{\mathbf{1}} \mathrm{D}$ Singles \\
\hline & 4 & & $\mathrm{~S}_{8} \mathrm{U}$ Singles \\
\hline & 5 & & $\mathrm{~S}_{\diamond} \mathrm{D}$ Singles \\
\hline & 6 & & $\mathrm{~S}_{8} \mathrm{U}$ Singles \\
\hline & 7 & & $\mathrm{~S}_{\triangleleft} \mathrm{D}$ Singles \\
\hline & 8 & & Beam Gates \\
\hline & 9 & & 1-MHz Clock \\
\hline & 10 & & $J_{1}+J_{2}$ \\
\hline & 11 & & \\
\hline \multirow[t]{6}{*}{$\# 4$} & 0 & \multirow[t]{6}{*}{ Run } & Left Telescope 12 (48 bits) \\
\hline & 2 & & Left Telescope 34 (48 bits) \\
\hline & 4 & & Left Telescope 1234 (48 bits) \\
\hline & 6 & & Right Telescope 12 (48 bits) \\
\hline & 8 & & Right Telescope 34 (48 bits) \\
\hline & 10 & & Right Telescope 1234 (48 bits) \\
\hline \multirow[t]{6}{*}{$\# 5$} & 0 & \multirow[t]{6}{*}{ Run $\cdot \overline{\text { Beam }}$} & $\mathrm{S}_{\mathrm{s}} \mathrm{U}$ Singles \\
\hline & 1 & & S,U Singles \\
\hline & 2 & & $\mathrm{~S}_{B} \mathrm{U}$ Singles \\
\hline & 3 & & $\mathrm{~S}_{\bullet} \mathrm{U}$ Singles \\
\hline & 4 & & Left Telescope 1 \\
\hline & 5 & & Right Telescope 1 \\
\hline \multirow[t]{4}{*}{$\# 6$} & 0 & \multirow[t]{4}{*}{ Run $\cdot$ Beam } & MWPC Accepted Triggers \\
\hline & 1 & & MWPC Rejected Triggers \\
\hline & 2 & & MWPC Read Errors \\
\hline & 3 & & MWPC No Plane Data \\
\hline \multirow[t]{4}{*}{$\# 7$} & 0 & \multirow[t]{4}{*}{ Run } & SEM1 \\
\hline & 1 & & SEM2 \\
\hline & 2 & & Ion Chamber 1 \\
\hline & 3 & & Ion Chamber 2 \\
\hline \multirow[t]{4}{*}{$\# 8$} & 0 & \multirow[t]{4}{*}{ Run } & Interrupt 3 \\
\hline & 1 & & SEM1 Duplicate \\
\hline & 2 & & Strobes \\
\hline & 3 & & Spare \\
\hline \multirow[t]{4}{*}{ \#9 } & 0 & \multirow[t]{4}{*}{ Run·Beam } & $\mathrm{C}_{\mathrm{L}} \mathrm{U}$ Singles \\
\hline & 1 & & $C_{L} D$ Singles \\
\hline & 2 & & $\mathrm{C}_{\mathrm{D}} \mathrm{U}$ Singles \\
\hline & 3 & & $\mathrm{C}_{\mathrm{D}} \mathrm{D}$ Singles \\
\hline
\end{tabular}




$\begin{array}{lcc}\text { Scaler } & \text { Channel } & \text { Gate } \\ \# 10 & 0 & \text { Run Beam } \\ & 1 & \\ 2 & \\ & 3 & \end{array}$

As an example of the use of thic system, consider a comparison of the first two scalers. The first scaler is inhibited when the computer is reading an event and the other scaler is not. The ratio of the two scaler sums is the fruction of time for which the CAMAC could not accept new events. Redundancy of several scaler channels with these two gates provides additional checks. Notice that the in. terrupts are also scaled in this way to provide a direct measurement of the events missed.

Another scaler type is the presettable countdown type of CAMAC scaler. Basically the computer can preset the number of counts to this scaler and after the scaler has counted that many pulses it produces a "Look-At-Me" signal (LAM) for the computer. Consider a hypothetical scattering experiment in which the data trigger is Interrupt 1 , with Interrupt 2 set investigate some background or event loss. By counting the Interrupt 2 events in this scaler system, the computer could be signaled to enable Interrupt 2 to form a strobe pulse and thereby obtain the Interrupt 1 data and simultaneously some predetermined sample of the Interrupt 2 correction data.

\section{APPENDIX D}

\section{COMPUTER INTERACTION WITH EXPERIMENT}

In addition to the ordinary functions of supervising data-taking, monitoring, and event sampling such as described in Appendix $\mathrm{C}$ a few remarks can be made about specific hardware aspects of the computer interaction with the experiment.

First, there is the matter of timing. It is probably not a good idea to assume that all CAMAC modules ignore additional strobe signals which arrive during encoding and readout. For this reason, the electronics design shown in Fig. 3 includes an LRS 222 gate generator which immediately inhibits the final coincidence for a time on the order of a few tens of microseconds. The Computer Busy signal, converted from TTL to NIM, is also sent to the gate generator's OR input. If the Busy signal arrives before the end of the initial inhibit, the inhibit remains for the duration of the Busy pulse.
Second, there is a finite digitizing time for many CAMAC modules with a typical commercial ADC module requiring $40 \mu 8$. One way to ensure that the operation is complete before reading is to test the module LAM and loop until the data are ready. With the microprugrammed branch driver (MBD) this is a difficult operation to limit, and if the module misses its strobe the entire data acquisition might be blocked. Another idea is to use the LAMPF Gate Generator to delay the strobe to the CAMAC interrupt module ${ }^{18}$ until the encoding is finished. The second method worked successfully but the interaction among the fast "strobe inhibit" pulse width, the delay chosen to allow encoding, and the scaler gate for "CAMAC Busy" will require careful consideration by the experimenter. 


\section{APPENDIX E}

\section{FAST, FOUR-CHANNEL DIFFERENTIAL DISCRIMINATOR}

\section{FUNCTION}

A fast, NIM discriminator having four independent channels is required with each channel having two threshold values, $\mathrm{th}_{1}$ and $\mathrm{th}_{2}$ with $0>\mathrm{th}_{1} \geq \mathrm{th}_{2}$. Three independent outputs should be available for each channel:

(1) NIM Output when signal $\geq$ th,

(2) NIM output when signal $\geq$ th $_{1}$ but $\leq$ th $_{2}$

(3) NIM output when signal $\geq t_{2}$, output timed by signal crossing at $\mathrm{th}_{1}$.

For computer control applications, the thresholds and widths for each channel should be remotely programmable. A rate capacity of $100 \mathrm{MHz}$ for both input and output is desirable with an input-output delay of $\leqslant 20 \mathrm{~ns}$. Also, the pulse-forming stage must be updating.

\section{SIGNAL INPUTS}

Dual thresholds should be capable of a range from $-30 \mathrm{mV}$ to $-10 \mathrm{~V}$, coupled so that $\mathrm{th}_{1} \geq \mathrm{th}_{2}$. Frontpanel screwdriver adjustments for each channel and threshold should be available. Fifty-ohm inputs should be protected to $\pm 5 \mathrm{~A}$ for $0.5 \mu \mathrm{s}$ with clamping at +1 and $-10 \mathrm{~V}$. Reflections $<5 \%$ for input pulses of 2-ns risetime. Stability $<0.5 \% /{ }^{\circ} \mathrm{C}$ over 20 to $60^{\circ} \mathrm{C}$ operating range. Maximum offset should be $0 \pm 5$ $\mathrm{mV}$.

Rear-connector inputs should provide threshold and width control for each channel with a 16-pin AMP or Burndy connector providing voltages from 0 to $-10 \mathrm{~V}$ with the amplitudes proportional to the width or thresholds desired.

\section{OUTPUTS}

Six normal NIM outputs ( $0 \mathrm{~mA}$ quiescently, -16 mA during output) per channel should be provided -2 each, mode $a, b$, and $c$. Pulse duration $\leqslant 6$ to $300 \mathrm{~ns}$, continuously adjustable from the front panel or programmable as above.

\section{PACKAgING}

Radiofrequency-shielded AEC/NIM \#1 modules with LEMO front-panel connectors.

\section{APPENDIX F}

\section{PATTERN COINCIDENCE/RECOGNITION MODULE}

\section{FUNCTION}

The module described here is intended for fast, on-line pattern "comparisons" between a single 16 bit word defined by external NIM electronics and a set of $4 \times 16$ bit words which are input manually or by computer. For each of these there will be an independent output strobe which becomes active following a valid comparison and remains so for a presettable time. The front-panel, NIM-level input word will accept inputs over the length of an input gate and the status of each bit is displayed by adjacent front-panel LEDs.

The four pattern words can be associated with four distinct event types or channels. Each channel has its own pattern to determine which of the bits must be set and which may be present to form a coincidence. In the pattern word a logical 1 would 
indicate that the presence of a signal is required for those bits. The remaining bits, at logical 0 , then determine whether the voter level specified by a control byte is satisfied. As a result, a coincidence output strobe for any channel requires that some number of signals be present in some inputs with the presence of all signals in the other set. However, the strobe may or may not be output depending upon whether a bit in the channel control byte is set or not.

The specifications given below are one possible means of achieving the above system. They were sent out for bid to essentially all commercial firms that might make such a module. We found that no such module was available and no substitutes were suggested.

\section{INPUTS}

16 Front-panel NIM inputs (LEMO) with $50 \Omega$ termination for input coincidence pulses.

1 Front-panel NIM input (LEMO) with $50 \Omega$ termination for input gate.

24-Bit, rear-connector, control injuut compatible with standard 24-bit CAMAC TTL output registers, consisting of a 16-bit coincidence word and an 8-bit control byte:

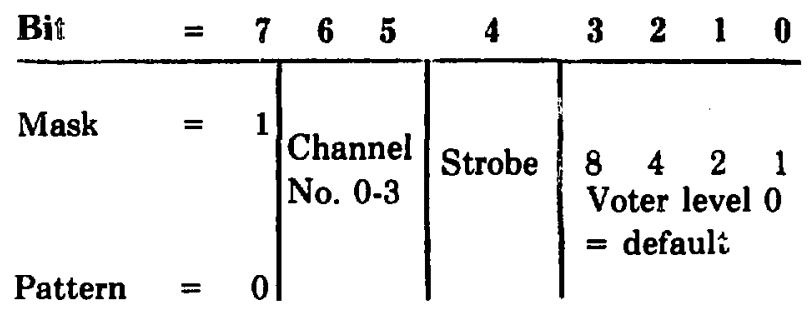

The coincidence word is either the Mask or Pattern as indicated in the control byte for a channel which is also selected in the control byte. Nonvolatile memory is desirable.

Front-panel, manual input and readout of the coincidence word and control byte should be available through selection of LOCAL on a local/computer switch. Readout should always be active in either mode.

The minimum input pulse widths should be $\geq 3$ ns, dc-coupled and overvoltage-protected. The delay between output strobe and input strobe is preferably $\leq 20$ to $30 \mathrm{~ns}$.

\section{OUTPUTS}

Two, fast, front-panel NIM outputs for each channel having individual width adjustments for each channel.

Twenty-four-bit rear-connector for readout to a remote location of the selected Mask or Pattern word in each channel.

\section{PACKAGING}

Double-width NIM.

\section{APPENDIX G \\ USE OF CHERENKOV COUNTERS FOR $(p, \pi)$ EXPERIMENTS}

A particularly good example of the use of Cherenkov counters is provided by the $(p, \pi)$ reaction because of the smallness of the $\pi / p$ ratio (typically $10^{-6}$ ). This is an instance where one ob- viously cannot accept a large sample with low selectivity and then let the computer do the final rejection analysis. The situation is further complicated by the large momentum acceptance (e.g., $25 \mathrm{MeV}$ ) 
and resolving power $\left(\geq 10^{4}\right)$ required to resolve discrete final levels at high excitation in the final nucleus. We will therefore assume that we cannot usf gas Cherenkov counters $\left(C_{G}\right)$ for specific elimination of electrons and that only plastic counters need be considered. Regardless, we know the light emission will be small

$\frac{\mathrm{dE}}{\mathrm{dx}} \sim 1 \mathrm{keV} / \mathrm{cm}$

and will vary with velocity. When one adds the requiremeni of total internal reflection, the lightcollection threshold becomes

$\beta \geq \sqrt{1 /\left(n^{2}-1\right)} n=1.49-0.905$.

This is shown in Fig. 13 as the left parenthesis for each particle type. After including the finite angular divergence of the beam near the focal plane and the energy losses with SLTSCT, it still follows that all heavy particles will be separated from $\pi, \mu$, and $e$ if we can eliminate knock-on electrons from the heavy particles and low efficiencies for the light particles. In heavy-particle experiments the Cherenkov counters would be used as a veto so that neither of these possibilities would be very important except possibly for $C_{D}$.

To be specific, consider the first value of momentum listed in Table $I$ at which pions are allowed ( $p$ $=665 \mathrm{MeV} / \mathrm{c}$ for $\mathrm{B}=6.336 \mathrm{~kg}$ ). Assuming that the Lucite counters are of sizes similar to $S_{3}$ and $S_{4}$ (Table II) implies an energy loss in the scintillator of 4.2 $\mathrm{MeV}$ for protons and $1.7 \mathrm{MeV}$ for pions with roughly 265 Cherenkov photons produced for the pions. Also, there is 2.36-ns/m TOF difference between them. Assumption of a $10 \%$ light-collection efficiency, $25 \%$ photoelectron response, and $60 \%$ light transmission gives (see Sec. III the following numbers of photoelectrons in scintillators:

$N_{p}^{5}=\frac{4.2 \times 10^{6}}{100} \times 0.1 \times 0.25 \times 0.60=630$

and

$N_{\pi}^{5}=255$.
For Cherenkov counters, the directional character of the light emission increases the light-collection efficiency by a factor of 2.5 so that

$\mathrm{s}_{\pi}^{\mathrm{e}}=10$

Obviously the Cherenkov counter will requi-e a discriminator setting just above single photoelectrons and will have photoeleitron fluctuations; therefore it is probably safest to operate it with two photomultipliers with an OR of their outputs. Additional precautions include measuring background rates to watch for photomultiplier gain fluctuations and monitoring high-voltage supplies closely. Operating at the kinematic limit of Fig. 13 does not offer improvement. Increasing the counter thickness would increase the light output proportionately, but the addition of significant amounts of material would also increase event losses from scattering.

Ka:ock-on electrons present a problem only for the heavy $p$-rrticles; they will tend to improve the Cherenkov response for the lighter ones. As a result we are concerned here about the probability of Cherenkov radiation from knock-on electrons produced mainly by protons. The electron spectrum will be forward-peaked ${ }^{10}$ and will depend on the amount of material upstream of the Cherenkov cou iter. The maximum energy given to an electron ${ }^{\circ}$ by a rarticle of rest mass $m$ is

$E_{\max } \approx 2 m e^{c^{2}(\beta \gamma)^{2}}$ for $\frac{\text { me }}{\pi} \ll \beta \gamma$

so that $E_{\max }=513 \mathrm{keV}$ from protons with $\beta_{\mathrm{p}}=0.578$ or a $\beta_{e} \leq 0.867$, which is below the light-collection threshold (compare to $\mathrm{E}_{\max }=23 \mathrm{MeV}$ for the pions). The energy loss of these electrons in plastic is $\sim 2 \mathrm{MeV} \mathrm{cral}^{2} / \mathrm{g}$ and the range is $\sim 0.18 \mathrm{~g} / \mathrm{cm}^{2}$ or only about $2 \mathrm{~mm}$ in plastic. Since all of our counters are thicker than this, the number of counters is of concern mostly for the multiple scattering out of the stack. However, in planning the layout of the Cherenkov counters and their logic for pions, one should take into account the $23 \cdot \mathrm{MeV}$ electrons.

If we use a lead absorber $\left(\rho=11.4 \mathrm{~g} / \mathrm{cm}^{2}\right)$ of $5.2+$ $0.2 \mathrm{~cm}$ thickriess it should stop the heavy particles 
and their knock-on electrons but still not reduce the pion below the light-collection threshold. For the case of $\mathrm{P} / \mathrm{z}=665 \mathrm{MeV} / \mathrm{c}$ :

\begin{tabular}{|c|c|c|c|}
\hline Particle & $\mathbf{T}$ & $\beta$ & $\begin{array}{c}\text { Pb Range } \\
\text { (cm) }\end{array}$ \\
\hline $\mathbf{P}$ & 211.7 & 0.578 & 5.2 \\
\hline$\pi$ & 539.8 & 0.979 & 38.2 \\
\hline e & 664.7 & 1.0 & 2.7 \\
\hline$\mu$ & 569.8 & 0.988 & 40.7 \\
\hline
\end{tabular}

But $5.2 \mathrm{~cm}$ is 0.53 colligion lengths, and a simpleminded expectation would be that $58.9 \%$ of $\pi$ and $p$ interact. Assuming that $\sim 1 / 4 \%$ of those protons produce neutrons which convert to "regenerate" a charged particle, $\sim 10^{-3}$ of protons may still be with us. For $\pi$, assume that half do elastic scattering and keep on going but that the inelastic ones are lost. This gives pion losses of about $.0 \%$ and will require investigation.

In summary, we have several alternatives, such as a counter array of $S_{2}, C_{L_{1}}, S_{3}, C_{L_{2}}, A_{1}, C_{D_{1}}$, $S_{4}, C_{D 2}\left(A_{2}, S_{b}, C_{L}, S_{A}, C_{D}\right)$ with a trigger based on $\left(C_{L 1} \cdot C_{L 2}\right) \cdot\left(C_{D 1} \cdot C_{T, 2}\right)$. Even though higher energy protons can refach the detectors following $A_{2}$, a longer flight path between $S_{2}$ and $S_{6}$ gives optimal time separation and greatly reduces their numbers. A detection efficiency for protons of $\leqslant 10^{-2}$ per counter for $C_{L 1}$ and $C_{L 2}$ is reasonable and is probably lower for $C_{D 1}$ and $C_{D 2}$ so that they can reduce the $p / \pi$ trigger ratio by as much as $10^{-8}$ or more while simultaneously providing a pion-detection efficiency of $\geq 0.4$ based on a conservative value of 0.8 per counter. The time separation is rather good, so it would be possible to take advantage of it in the fast trigger electronics (particularly with use of the LRS Mean 'imers) without use of $8 \mathrm{C}$-ns chopped beam so that th OR options could be used for both pairs of Cherenkov counters, except when we want to measure cross sections $\leqslant 0.1 \mathrm{nb} / \mathrm{sr}$ at some angles. With all these possibilities, the use of the multiplestrobe feature of Fig. 3 is suggested as well as simulation of the final setup with SLTSCT to calculate the effect of the nuclear reactions in the degrader on the Cherenkov response. Note that with this setup we could run with average rates higher then $10 \%$ s sn the scintillators and still obtain good data so long as we don't have to rely on timing to separate events.

\begin{tabular}{|c|c|c|c|}
\hline Lo8s & B after & $r_{c}$ before & $\gamma_{c}$ after \\
\hline 211.7 & 0 & (knock-on?) & 0 \\
\hline 98.0 & 0.971 & 265 & 261 \\
\hline 664.7 & 0 & 275 & 0 \\
\hline 71.1 & 0.976 & 269 & 263 \\
\hline
\end{tabular}

\section{REFERENCES}

1. A complete layout, including all devices, is given in Las Alamos Scientific Loboratory DWG 75Y174083E-3.

2. K. L. Brown, "A First- and Second-Order Matrix Theory for the Design of Beam Transport Systems and Charged Particle Spectrometers," Stanford Linear Accelerator Center Report 75 (1967).

3. D. Brown, A. Greenwood, J. C. Pratt, J. E. Spencer, "Multichannel Remotely Programmable Logic Delay System," Nucl. Instrum. Methods 138, 695 (1976).

4. R. G. Clarkson and N. Jarmie, "Energy-Loss Straggling of Heavy Charged Particles," Computer Phys. Commun. 2, 433 (1971).

5. Program SLTSCT, P. C. Gugelot, J. C. Pratt, and J. E. Spencer (Los Alamos Scientific Laboratory). Writeup is available from authors.

6. L. C. Northcliffe, "Passage of Heavy Ions Through Matter," Ann. Rev. Nucl. Sci. 13, 67 (1963).

7. D. A. Gedcke and C. W. Williams, "High Resolution Time Spectroscopy," ORTEC Inc., Oak Ridge, TN, Special Publications, 1968.

8. W. J. McDonald and D. A. Gedcke, "Time Resolution Studies on Large Photomultip!iers," Nucl. Instrum. Methods, 55, 1 (1967). 
9. Vladimir Chaloupka et al., "Review of Particle Properties," Phys. Lett. 50B, 1-193 (1974).

10. J. K. Walker, "Characteristics of Plastic Scintillators in Their Use as Large Area Particle Detectors," Nucl. Instrum. Methods 68, 131 (1954).

11. G. Igo and R. M. Eisberg, "Use of Proportional Counters in the Measurement of the Specific Ionization of Charged Particles," Rev. Sci. Instruns. 25, 450 (1954);

K. Nagata, "A Semiconductor Detector Telescope for Identifying Mass or Charge of High Energy Particles Based on the Minimum Pulse Height Selection Method," Nucl. Instrum. Methods 77, 218 (1970).
12. G. Charpak, L. Dick, and L. Feuvrais, "Location of the Position of a Particle Trajectory in a Scintillator," Nucl. Instrum. Methods 15, 323 (1962).

13. W. K. McFarlane, "An Inexpensive Nanosecond Light Pulser for Use in Photomultiplier System Testing," Rev. Sci. Instrum. 45, 236 (1974).

14. A. P. Conley, "IAMPF Gate Generator," LAMPF Internal Report, 1972.

15. I. D. Nickell, "LAMPF Event Trigger Module," LAMPF Internal Report, 1975.

16. B. Rossi, High Energy Particles, (Prentice Hall, Englewood Clifis, N.J., 1961). 


\section{GLOSSARY}

$\begin{array}{ll}\text { CCH } & \text { Area counting house } \\ \text { HRS } & \begin{array}{l}\text { high regolution spectrometer } \\ \text { "look-at-men signal }\end{array} \\ \text { LAM } & \text { iight-emitting diode } \\ \text { MED } & \text { microprogrammed branch driver } \\ \text { MWPC } & \text { multiwire proportional chamber } \\ \text { PIDS } & \text { particle-identification system } \\ \text { QDMD } & \text { quadrupole-dipole-multipole-dipole } \\ \text { SEM } & \text { secondary emisgion monitor } \\ \text { TDC } & \text { time-to-digital converter } \\ \text { TOF } & \text { time of flight } \\ \text { UVT } & \text { ultraviolet-transmitting }\end{array}$

